\title{
Cluster observations of a transient signature in the magnetotail: implications for the mode of reconnection
}

\author{
S. Beyene ${ }^{1}$, C. J. Owen ${ }^{1}$, A. P. Walsh ${ }^{1}$, C. Forsyth ${ }^{1}$, A. N. Fazakerley ${ }^{1}$, S. Kiehas ${ }^{2,3}$, I. Dandouras ${ }^{4,5}$, and E. Lucek ${ }^{6}$ \\ ${ }^{1}$ Mullard Space Science Laboratory, University College London, Holmbury St. Mary, Dorking, RH5 6NT, UK \\ ${ }^{2}$ Space Research Institute, Austrian Academy of Sciences, Schmiedlstrasse 6, 8042 Graz, Austria \\ ${ }^{3}$ Institute of Geophysics and Planetary Physics, University of California, Los Angeles, California, USA \\ ${ }^{4}$ Institut de Recherche en Astrophysique et Planétologie (IRAP), University of Toulouse, UPS, 9 Ave. du Colonel Roche, \\ B.P. 44346, 31028 Toulouse, France \\ ${ }^{5}$ IRAP, CNRS, B.P. 44346, 31028 Toulouse, France \\ ${ }^{6}$ Space and Atmospheric Physics, Imperial College London, SW7 2BZ, UK
}

Received: 17 August 2011 - Revised: 18 October 2011 - Accepted: 27 October 2011 - Published: 28 November 2011

\begin{abstract}
Travelling compression regions (TCRs) are perturbations in the magnetotail lobe magnetic field caused by structures moving Earthward or tailward within the plasma sheet. Previous works have suggested that these structures are created by either time-dependant reconnection occurring at a single X-line, forming a flux-bulge-type structure, or space-variant reconnection at multiple $\mathrm{X}$-lines, forming fluxrope-type structures. In this study we examine an event in which Cluster 2 observed a TCR while the 3 remaining Cluster spacecraft observed the underlying magnetic structure at a range of distances from the neutral sheet. The magnetic structure has a velocity of $(99,154,-31) \mathrm{km} \mathrm{s}^{-1}$ in GSM $\left(|\underline{V}|=186 \mathrm{~km} \mathrm{~s}^{-1}\right)$, an estimated size of $1.19 R_{\mathrm{E}}$ along the direction of travel and a size between 1.94 and $2.86 R_{\mathrm{E}}$ in the direction perpendicular to the current sheet. As the structure passes the spacecraft, Cluster 1 and Cluster 4 observed a bipolar signature in $B_{Z}$, plasma-sheet-like plasma and fieldaligned electron flows. Cluster 3 passed closest to the centre of the structure and observed two separate reductions in the plasma density (with field-aligned electron flows); these drop-outs in the plasma sheet were possibly created by the actions of $\mathrm{X}$-lines. The second drop-out in the plasma sheet also includes a reversal of the ion flow, a signature consistent with the passage of a reconnecting $\mathrm{X}$-line past the spacecraft. Between the X-lines, the plasma outflow from the X-lines caused an increase in pressure which led to a localised expansion of the plasma and also the observations at Cluster 1
\end{abstract}

Correspondence to: S. Beyene

(sb2@mssl.ucl.ac.uk) and Cluster 4 and the TCR. Our observations do not uniquely match either of the flux rope or the flux bulge predictions although the observation of two plasma sheet drop-outs (interpreted as X-lines, one active, one dormant) with plasmasheet-like between them and only one TCR is a situation expected in multiple $\mathrm{X}$-line reconnection.

Keywords. Magnetospheric physics (Magnetotail; Plasma sheet) - Space plasma physics (Magnetic reconnection)

\section{Introduction}

Travelling Compression Regions (TCRs) (Slavin et al., 1992, 2003b, 2005; Owen et al., 2005) are transient perturbations and compressions of the magnetotail lobe. The compression of the lobe in the direction perpendicular to the current sheet results in a peak in the magnetic field magnitude (and hence $B_{X}$ GSM as the tail field is directed mostly in X GSM) and a bipolar signature in the magnetic component directed normal to the current sheet (nominally in Z GSM). Observations have shown that TCRs have a compression ratio $(\Delta B / B)$ of $\sim 1-10 \%$ (Slavin et al., 2005). TCRs have been observed travelling both Earthwards and tailwards; the direction of travel can be deduced from the sense of the bipolar signature. A bipolar signature consisting of a negative excursion followed by a positive excursion in $B_{Z}$ has been shown to be Earthward travelling (Slavin et al., 2003a); conversely, bipolar signatures consisting of a positive excursion followed by a negative excursion are tailward travelling.

Published by Copernicus Publications on behalf of the European Geosciences Union. 
Previous studies have suggested that TCRs are caused by magnetic structures created in the near-Earth magnetotail (Elphic et al., 1986; Moldwin and Hughes, 1994; Slavin et al., 2003a; Zong et al., 2004). Observations of Earthward travelling magnetic structures by Slavin et al. (2003a) identified 35 bipolar signatures, all of which were located between -10 and $-30 R_{\mathrm{E}}$ (X GSM). These structures are formed by reconnection (Elphic et al., 1986; Hughes and Sibeck, 1987), and are centred on the current sheet. However, it is still not clear whether these structures are a result of time-varying reconnection at a single reconnection site (e.g. Semenov et al., 1983, 1984; Biernat and Heyn, 1987) or reconnection occurring at multiple reconnection sites within the tail (e.g. Slavin et al, 2003a; Walsh et al., 2007).

In this paper, we examine a magnetic structure observed in the magnetotail by the Cluster spacecraft during a period when the relative position of the 4 spacecraft allowed the structure and its environs to be variously sampled. We attempt to deduce which, if either, of the two possible modes of reconnection more closely matches the observations and hence which was most likely responsible for its formation. The structure of the paper is as follows: Sect. 2 reviews the predictions of the time-varying and space-varying reconnection models; Sect. 3 details the instruments providing data for this study, together with the orbit and configuration of the spacecraft; Sect. 4 presents the observations; Sect. 5 is an analysis of the data; Sect. 6 provides an interpretation of the data and discusses the possible explanations and Sect. 7 notes the conclusions.

\section{Review of models of magnetotail reconnection}

\subsection{Multiple X-line Reconnection (MXR)}

The original model for multiple reconnection sites was proposed by Lee and $\mathrm{Fu}$ (1985) in the context of the dayside magnetopause, and assumes that reconnection occurs at two (or more) adjacent reconnection sites (hereafter referred to as $\mathrm{X}$-lines). These will create a magnetic loop between them, nominally lying in the XZ GSM plane (in the case of magnetotail MXR). Hughes and Sibeck (1987) showed that, if there is a cross-tail magnetic field (originating from a $B_{Y}$ component of the IMF), this mode of reconnection will create a helical magnetic structure, known as a flux rope (Elphic et al., 1986; Slavin et al., 2003a). One of the multiple X-lines within the tail will generally reconnect faster than the others (Schindler, 1974), and will thus begin to reconnect open lobe magnetic flux prior to other X-lines. Lobe field lines reconnected at this "dominant" X-line will subsequently envelope the set of flux ropes forming on either side. As illustrated in Fig. 1, these hairpin-like field lines subsequently act to expel the plasma and the magnetic structures away from the dominant X-line (Schödel et al., 2001; Slavin et al., 2003a) along the current sheet; this occurs on either side of the X-

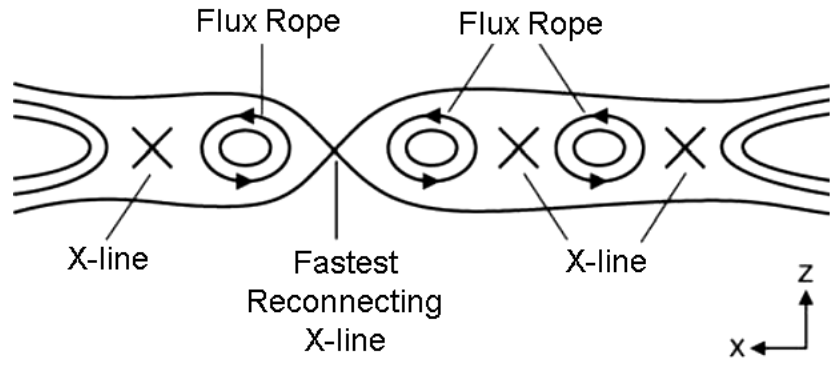

Fig. 1. Diagram of the central region of the magnetotail showing multiple X-lines and the flux ropes that form between them. The newly reconfigured, kinked field lines that envelope the flux ropes act to expel them Earthward and tailward away from the fastest reconnecting X-line. Diagram adapted from Slavin et al. (2003a).

line. Plasma, reconnected field lines and flux ropes located Earthward (tailward) of the dominant X-line, travel in the Earthward (tailward) direction.

The simplest model of the structure of a magnetic flux rope is the force-free model (e.g. Lepping et al., 1990), which is the minimum energy configuration for helical magnetic fields. The model is force-free $(\underline{F}=\underline{J} \times \underline{B}=0)$ as the magnetic tension force acting radially inwards is balanced by the outward-directed magnetic pressure force, such that the structure will not evolve with time (Priest, 1990). Forces associated with the presence of plasma are ignored in this model. The current is parallel to $B$ everywhere, $\underline{J}=\alpha \underline{B}$, and "constant $\alpha$ " solutions for such structures are cylindrical, with the magnetic field largely azimuthal at the outer edge and becoming increasingly axial towards the centre, often resulting in a strong core field. The core field of plasma sheet flux ropes can be up to twice the intensity of the tail lobes (Slavin et al, 1995). The structure is invariant along the cylinder axis (hereafter referred to as the invariant axis).

Figure 2 shows the variation of the components of the magnetic field expected to be observed by a spacecraft passing through the centre of an idealised Earthward-travelling force-free flux rope. For a crossing through the centre of a flux rope, a spacecraft will detect a $B_{X}$ of zero, a unipolar $B_{Y}$ and $|B|$ signature and a bipolar $B_{Z}$ signature. An off-centre crossing (i.e. a crossing with a non-zero impact parameter), is similar to the centre crossing with the exception that $B_{X}$ will also peak at closest approach. With increasing impact parameter, the magnitude and duration of the $B_{Y}, B_{Z}$ and $|B|$ signatures will decrease. The bipolar $B_{Z}$ signature will have a positive and negative excursion of similar magnitude.

Due to the compression in the lobe caused by the flux rope, a spacecraft in the lobe will detect a TCR (Slavin et al., 2003b). A spacecraft travelling through a flux-ropegenerated TCR will detect a $B_{X}$ and $|B|$ signature that peaks at closest approach, a $B_{Y}$ signature that depends on the IMF induced $B_{Y}$ in the tail lobe and a bipolar $B_{Z}$ signature. The bipolar signature will have excursions of similar magnitude and duration. During a TCR, the $B_{X}$ component and $|B|$ will 


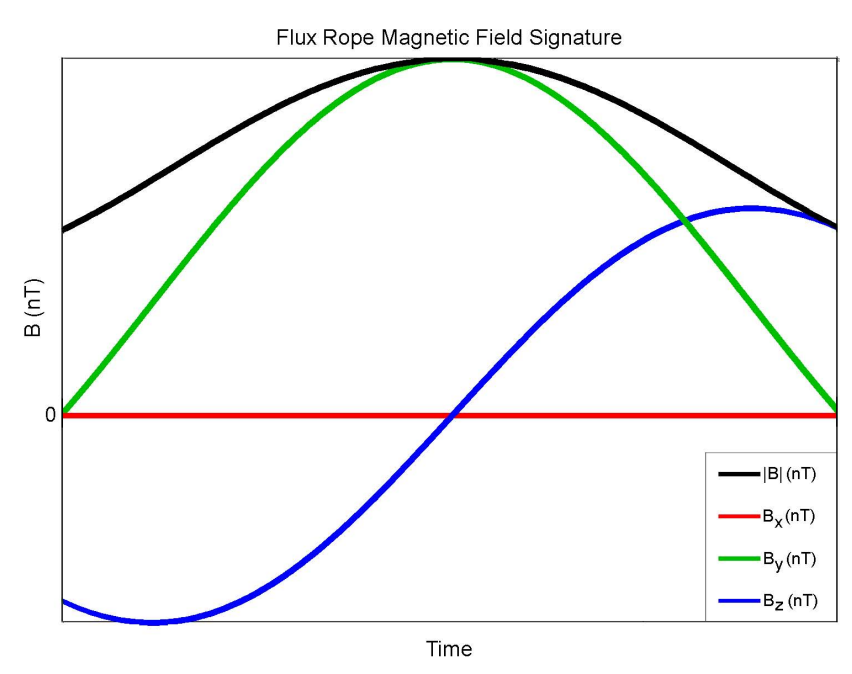

Fig. 2. Diagram of the magnetic field signature of a virtual spacecraft travelling through the centre of an Earthward travelling force free model of the flux rope. The figure shows a plot of $B_{X}$ (red), $B_{Y}$ (green), $B_{Z}$ (blue) and $|B|$ (black) vs. time. The $B_{X}$ trace is zero, the $B_{Y}$ and $|B|$ traces peak and the $B_{Z}$ trace shows a bipolar signature. The bipolar magnetic signature of the force free flux rope has excursions of similar magnitude and duration.

peak, before reducing to a level lower than the pre-encounter value. This is a result of reconnection driving a net loss of magnetic flux from the tail in association with the formation of the flux rope. Flux ropes have been directly observed and reported by a number of authors (e.g. Slavin et al., 1999, 2003a; Walsh et al., 2007; Imber et al., 2011), they are 2$5 R_{\mathrm{E}}$ in lateral extent and likely to be consistent with the $\Delta B / B \sim 1-10 \%$ values reported in the literature.

Non-force-free magnetic structures have also been observed (e.g. Henderson et al., 2006) and more sophisticated models have been created to account for them (e.g. Mulligan and Russell, 2001). The implied imbalance of internal magnetic forces suggests such structures may be undergoing an evolution towards a force-free magnetic structure as this is the minimum energy configuration. Real flux ropes are expected to contain plasma; if a plasma gradient is present, this will contribute plasma pressure forces to the overall force balance. The presence of plasma in a flux rope will generally decrease the magnetic field strength below that of an ideal force free flux rope due to the diamagnetic effect. We are unaware of any studies of the characteristics of particle populations inside magnetotail flux ropes. Due to the concentricity of the field lines of a flux rope, the distribution of the plasma may be expected to be spatially symmetrical about the axis, and thus also symmetric about the inflexion point of the bipolar signature in observations from spacecraft passing through such structures. As the flux rope is formed in the central plasma sheet (CPS), it is expected to contain CPS-like plasma.

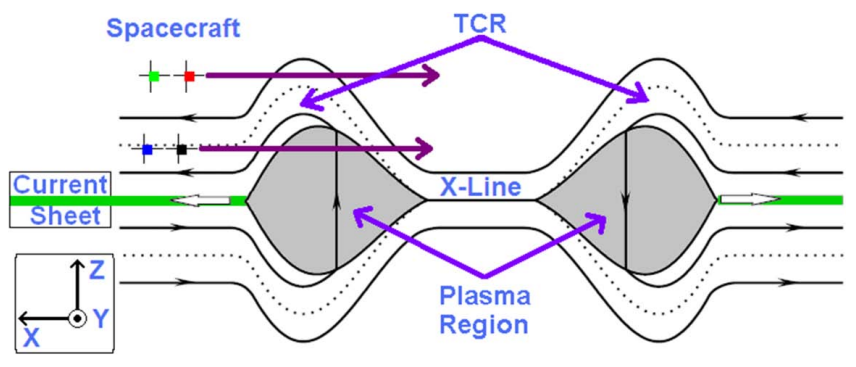

Fig. 3. A representation of the central magnetotail and the flux bulges resulting from time-dependant reconnection (adapted from Kiehas et al., 2009). The heated and accelerated plasma forms a tear-drop-shaped outflow plasma region which displaces the lobe magnetic field lines around it, causing a TCR. The reconnected field lines, which are also displaced, run through the outflow plasma region and the current sheet. Throughout the outflow plasma region of an Earthward travelling flux bulge, the magnetic field has a northward component.

\subsection{Time-dependent reconnection model}

Semenov et al. (1983, 1984) and Biernat and Heyn (1987) developed a model of magnetic reconnection based on Petscheck-type reconnection (Petscheck, 1964) at a single $\mathrm{X}$-line. In this model, a time-varying electric field implies a time-varying reconnection rate. A diagram of the resulting structure and the expected observed magnetic signature are depicted in Fig. 3. In this model, reconnection creates a set of standing shocks which bound the plasma outflow region. Moreover, the variation in reconnection rate creates a tear-drop, or bulge-shaped, plasma outflow region; the magnetic field within this region is relatively weak, due to the diamagnetic effect of the heated plasma downstream of the shocks. The reconnected magnetic field and plasma region will be collectively referred to here as a flux bulge. In this model the magnetic field in the heated plasma region is directed perpendicular to the current sheet. Figure 3 also shows that the outer field lines (furthest from the current sheet) are draped around the heated plasma region causing a TCR-type signature. Field lines initially lying closer to the current sheet are partially draped around the heated plasma region but also thread through the plasma region and the embedded current sheet. When the electric field disappears, reconnection ceases; the flux bulges detach from the site where reconnection was initiated and the reconnected field lines expel the flux bulges in opposite directions away from the X-line, along the current sheet.

As in the MXR model, the passage of a flux bulge will result in a spacecraft in the lobe detecting a TCR signature, as shown in Fig. 3, taken from Kiehas et al. (2009). The amplitude of the TCR will depend on the size of the flux bulge but this is likely to be consistent with the $\Delta B / B \sim 1-10 \%$ values reported in the literature. For the flux-bulge-generated TCR, the $B_{X}$ component and $|B|$ will peak, before reducing to a 
level lower than the pre-encounter value (for the same reason given in MXR) and the $B_{Y}$ component (not shown) will depend on the IMF induced $B_{Y}$ in the tail. The $B_{Z}$ component will contain a bipolar signature; an Earthward-travelling flux bulge will show a south-north bipolar signature with a smaller (in magnitude and duration) negative excursion in comparison to the positive excursion. A tailward-travelling flux bulge will have a north-south bipolar signature with a smaller (in magnitude and duration) positive excursion.

For trajectories lying closer to the current sheet, the spacecraft will encounter the heated plasma region itself. Along these trajectories, whilst outside of the plasma region, the spacecraft will detect part of the TCR signature. The negative excursion of the bipolar signature will be detected prior to entry into the plasma region. Within the plasma region, the spacecraft will detect a very low $B_{X}$ and a peak in $B_{Z}$. For a central crossing (a spacecraft travelling along the current sheet), the only signature detected is the peak in $B_{Z}$. Unlike the flux rope predictions, flux bulges are not expected to have a strong core field; instead the heated plasma of the plasma region reduces the magnetic field strength due to the diamagnetic effect (to below the lobe field strength). As the flux bulge is formed in the CPS, the plasma region is expected to contain CPS-like plasma.

\section{Instrumentation}

The data used in this paper come from the instruments of the 4 ESA Cluster spacecraft (Escoubet et al., 2001).

Data on the magnetic field vector at each spacecraft are collected by the Fluxgate Magnetometer (FGM) (Balogh et al., 2001), which provides 3-D magnetic field vectors at a frequency of up to $67 \mathrm{~Hz}$; here we use $5 \mathrm{~Hz}$ data.

The ion moments and velocity distribution data are returned from the Cluster Ion Spectrometer (CIS) data (Rème et al., 2001), which consists of two different sensors; the Hot Ion Analyser (HIA) and the time-of-flight ion Composition Distribution Function Analyser (CODIF). HIA has an energy/charge ratio in the range of $\sim 5 \mathrm{eV} \mathrm{q}^{-1}-32 \mathrm{keV} \mathrm{q}^{-1}$. CODIF can distinguish ions by mass and has an energy/charge ratio in the range of $\sim 15 \mathrm{eV} \mathrm{q}^{-1}-38 \mathrm{keV} \mathrm{q}^{-1}$. CIS data is not available from either HIA or CODIF on C2 nor from HIA on $\mathrm{C} 4$. Observations for $\mathrm{C} 1$ and $\mathrm{C} 3$ in this paper were made by HIA and observations of $\mathrm{C} 4$ by CODIF. During the observations presented here, HIA on $\mathrm{C} 1$ and $\mathrm{C} 3$ had a resolution of $4 \mathrm{~s}$ and CODIF on $\mathrm{C} 4$ had a time resolution of $8 \mathrm{~s}$.

The data presented here have been corrected for known instrumental effects.

The electron moments and distribution data were obtained from the Plasma Electron And Current Experiment (PEACE) instrument (Johnstone et al., 1997). PEACE measures the 3$D$ velocity distribution function of electrons in space plasma, for an energy range from $\sim 0.6 \mathrm{eV}$ to $\sim 26.4 \mathrm{keV}$. During the

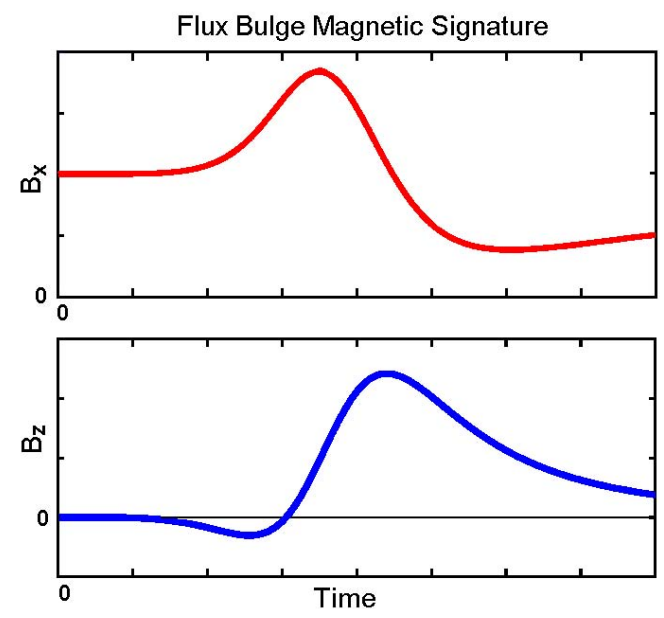

Fig. 4. Modelled magnetic signature of a virtual spacecraft passing through the flux bulge TCR (the magnetic field draped over an Earthward travelling flux bulge; diagram taken from Kiehas et al., 2009). The plot shows $B_{X}$ (red trace) peaks and reduces to a level lower than the initial level. $B_{Z}$ (blue trace) shows a bipolar signature with a negative excursion smaller in magnitude and duration than the positive excursion. A spacecraft with a trajectory through the plasma region will detect the negative excursion of the bipolar signature prior to entry. Within the plasma region, the spacecraft will detect little $B_{X}$ and a peak in $+B_{Z}$. A central crossing (along the current sheet) will only detect a $+B_{Z}$ peak. The heated outflow plasma has a reduced magnetic field strength due to the diamagnetic effect.

observations, PEACE on all four spacecraft had a time resolution of $4 \mathrm{~s}$.

All of the plasma moments data presented in this paper are derived from CIS data, except for those form C2 (for which CIS data is unavailable); PEACE moments are used in its place. The FGM, CIS and PEACE data were all obtained from the Cluster Active Archive except for the C4 CODIF data which was supplied directly by the CIS team after instrumental artifacts had been removed.

In order to obtain the most complete picture of the disturbance causing the TCR (dubbed here the "magnetic structure"), we actively seek events for which the spacecraft separations are close to the expected size of the magnetic structure itself. This provides measurements of the passing structure from the multiple Cluster spacecraft which are then likely to have a good spread of impact parameters. In the case of Earthward-moving structures, these are typically reported as being $2-5 R_{\mathrm{E}}$ in diameter (e.g. Slavin et al., 2003a). Cluster separations best fulfil this requirement during the 2006 and 2007 tail seasons (July-November each year), during which the typical separations between spacecraft were of the order of $10000 \mathrm{~km}$. In this paper, we report results from a case study of observations made between 04:40-04:45 UT on 7 October 2006, at which time the individual spacecraft clearly sampled different parts of a passing magnetic 

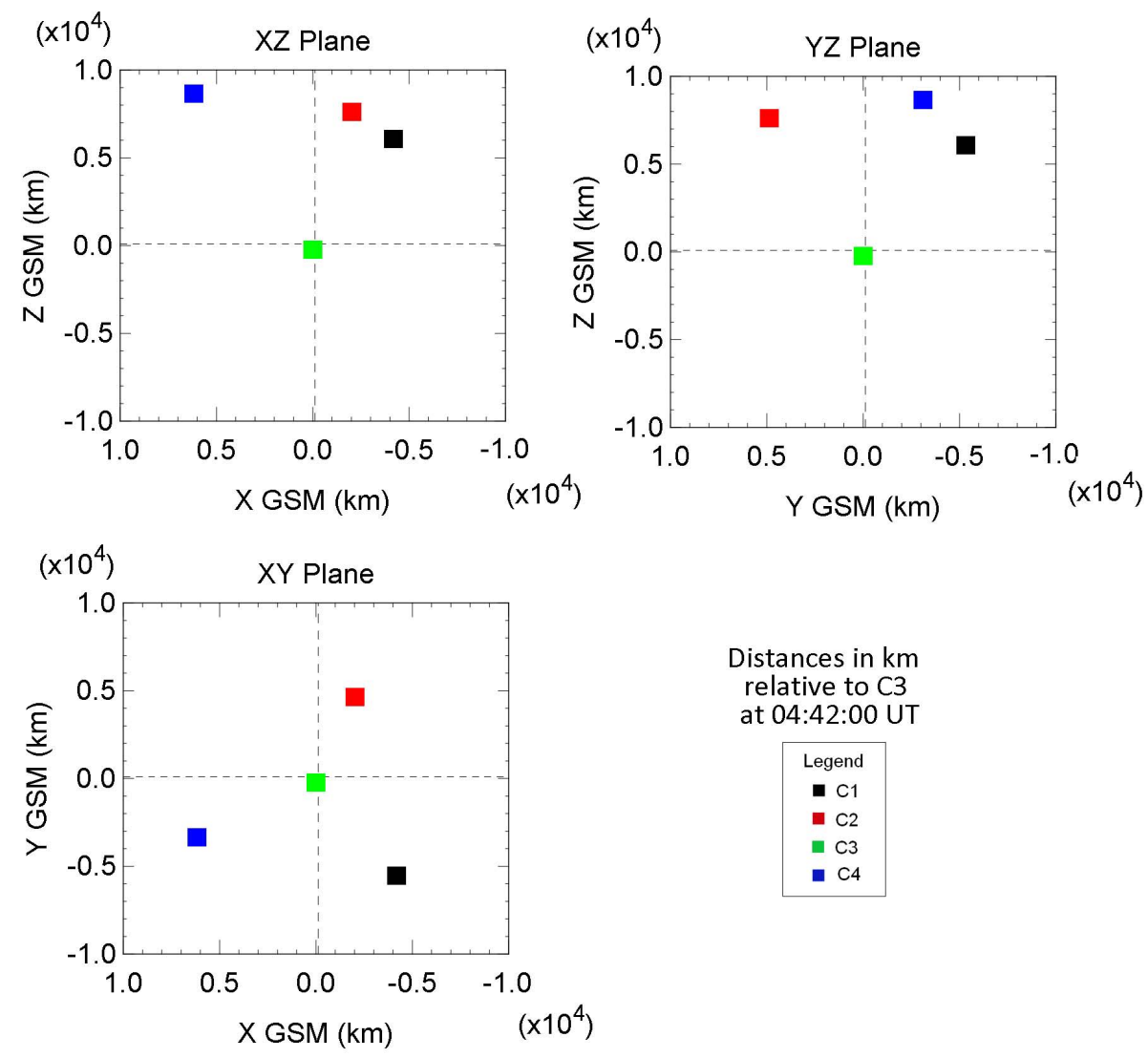

Fig. 5. Spacecraft position relative to $\mathrm{C} 3$ at 04:42:00 UT on 7 October 2006 in GSM. The top left panel shows the $X Z$ plane, the top right shows the YZ plane and the bottom left shows the $\mathrm{XY}$ plane. $\mathrm{C} 1$ is represented by the black square, $\mathrm{C} 2$ is red, $\mathrm{C} 3$ is green and $\mathrm{C} 4$ is blue. The spacecraft were in the magnetotail in an approximate tetrahedral formation with $\mathrm{C} 3$ lower than the other spacecraft in $\mathrm{Z}$ by $\sim 6000 \mathrm{~km}$. $\mathrm{C} 1, \mathrm{C} 2$ and $\mathrm{C} 4$ were within $3000 \mathrm{~km}$ of each other in $\mathrm{Z}$.

structure. The spacecraft were located within the magnetotail, with $\mathrm{C} 3$ at $(-13.97,5.11,-1.92) R_{\mathrm{E}}$ at $04: 42 \mathrm{UT}$ (the coordinate system used in this paper is GSM unless stated otherwise). The positions of the spacecraft relative to $\mathrm{C} 3$ are illustrated in Fig. 5. The three panels show the relative spacecraft locations in the XY, XZ and YZ planes. The four spacecraft were in a near regular tetrahedral formation with C3 $\sim 6000 \mathrm{~km}$ south of the other spacecraft, while C1, C2 and $\mathrm{C} 4$ were within $3000 \mathrm{~km}$ of each other in $\mathrm{Z}$.

\section{Observations}

Figure 6 shows the magnetic field and the PEACE electron data recorded between 04:15-05:15 UT on 7 October 2006. The top 4 panels each show the $B_{X}$ (red trace), $B_{Y}$ (green trace) and $B_{Z}$ (blue trace) components of the magnetic field, together with the field magnitude (black trace), for each of the 4 spacecraft, $\mathrm{C} 1$ to $\mathrm{C} 4$, respectively. The lower 4 panels show spectrograms of direction-averaged electron differential energy flux (DEF) over the PEACE energy range versus time at each spacecraft. Initially, all four spacecraft were lo- cated in a region of strong and steady magnetic field (directed in positive $B_{X}$ ) with low particle DEF. This region is identified as the lobe. Between 04:30 and 04:44 UT, C1 observes a similar magnetic field strength and negligible particle DEF indicating that it is in the northern tail lobe. At $\sim 04: 38 \mathrm{UT}$, $\mathrm{C} 3$ entered the central plasma sheet (CPS); the region is characterised by a relatively weak and more variable magnetic field and relatively enhanced plasma DEF, especially evident at higher energies. At 04:41-04:42 UT, C1, C2 and C4 detected a set of bipolar signatures in $B_{Z}$.

We now consider the first, largest and most significant bipolar signature which is observed between 04:4004:45 UT (indicated by the vertical lines in Fig. 6), in more detail and on a spacecraft-by-spacecraft basis. These are presented in the order $\mathrm{C} 2, \mathrm{C} 4, \mathrm{C} 1$ and $\mathrm{C} 3$, which, on the basis of the data described above, we believe reflects the order of decreasing impact parameter.

Over a longer period of time (not shown), the plasma sheet appears to be "flapping" (e.g. Sergeev et al., 2008; Zhang et al., 2002; Runov et al., 2005; Forsyth et al., 2009). This is the oscillatory motion of the plasma sheet in the direction perpendicular to the current sheet. However, as the period 


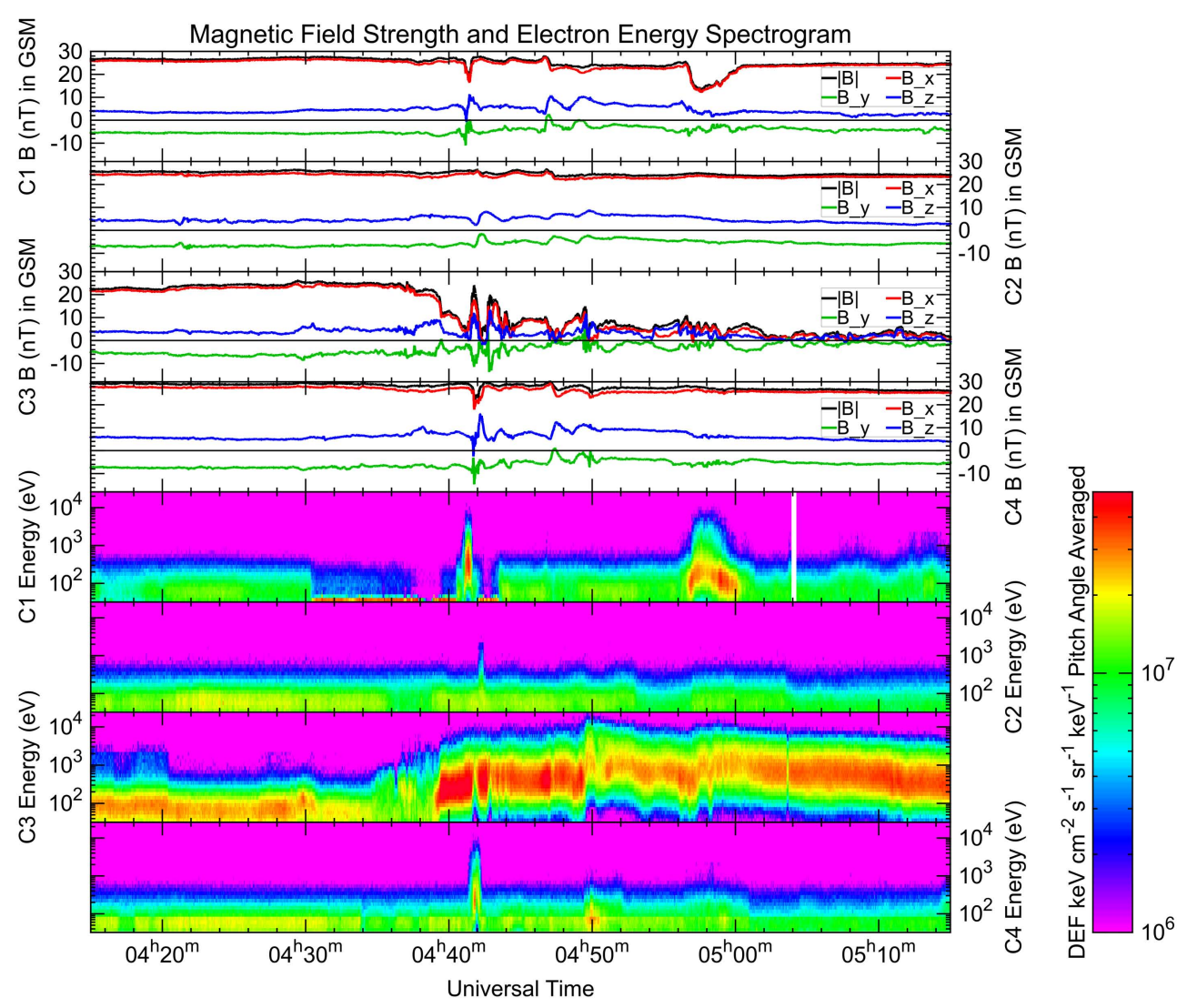

Fig. 6. Panels 1, 2, 3, 4 shows the magnetic field strength for $\mathrm{C} 1, \mathrm{C} 2, \mathrm{C} 3, \mathrm{C} 4$ respectively $\left(B_{X}\right.$ is in red, $B_{Y}$ is in green $B_{Z}$ is in blue and $|B|$ is in black). Panel 5, 6, 7, 8 show the (pitch angle averaged) electron energy spectrogram of $\mathrm{C} 1$, C2, C3, C4, respectively. Between 04:40-04:50 UT, C1, C2 and C4 encountered a set of bipolar signatures in $B_{Z}$, the largest of which was detected between 04:40-04:45 UT, marked by the two dark vertical lines. Coinciding with the bipolar signature, $\mathrm{C} 1, \mathrm{C} 2$ and $\mathrm{C} 4$ detected an increase in electron DEF. Between the marked times, $\mathrm{C} 1, \mathrm{C} 2$ and $\mathrm{C} 4$ were in a region of a large steady $B_{X}$ and low electron DEF which indicates they are outside the central plasma sheet. C3 was in a region of low, varying $B_{X}$ with a high electron DEF which indicates it is inside the central plasma sheet.

of the oscillation $(\sim 30 \mathrm{~min})$ is much greater than the time scale of the signature of interest ( $\sim 5 \mathrm{~min})$, it seems unlikely that the latter may arise as a manifestation of the former and hence the possibility is ruled out.

Figure 7 shows the data from $\mathrm{C} 2$ for the period 04:4004:45 UT. Panel 1 shows the magnetic field strength and GSM components $\left(B_{X}, B_{Y}, B_{Z}\right.$ and $|B|$ are in red, green, blue and black, respectively). Panel 2 and 3 respectively show an electron energy spectrogram (pitch-angle-averaged) and an electron pitch angle spectrogram (energy-averaged) of the observed DEF (in units of $\mathrm{keV} /\left(\mathrm{cm}^{2} \mathrm{~s}\right.$ sr keV)). Panels 4-7 show electron density, electron temperature, electron $\beta$ and electron perpendicular velocity, respectively. Across the whole time period, $\beta<0.3$ which indicates that the spacecraft is outside of the central plasma sheet (Baumjohann et al., 1989) and is hence in the lobe. At 04:42 UT, the observations indicate that $\mathrm{C} 2$ observed a clear negative-positive bipolar signature in the $B_{Z}$ component, with a peak-to-peak amplitude of $\sim 6 \mathrm{nT}$. The dotted vertical lines mark the turning points of this bipolar signature. Simultaneously, the $B_{Y}$ component changed from $\sim-7 \mathrm{nT}$ to $\sim-2 \mathrm{nT}$, while $B_{X}$ and $|B|$ show much smaller variations. The duration of the bipolar signature (measured from peak to peak) is $\sim 31 \mathrm{~s}$, covering 156 magnetic field data points and 8 plasma data points. Each of the magnetic field traces in Fig. 7 show a generally smooth profile to the variations. During the bipolar magnetic signature, there is also a slight increase in the electron DEF at energies over $3 \mathrm{keV}$ at all pitch angles which is greatest for $0^{\circ}$ and $180^{\circ}$. The increase in DEF is also reflected in the concurrent increase in the electron density and electron temperature. Moderate flows are seen in the perpendicular velocity; $V_{\perp X}$ shows only a small peak and $V_{\perp Z}$ shows a north-south bipolar signature.

Figure 8 shows the data from $\mathrm{C} 4$. Panel 1 shows the magnetic field strength and GSM components $\left(B_{X}, B_{Y}, B_{Z}\right.$ and $|B|$ are in red, green blue and black, respectively). Panels 2-5 respectively show a proton energy spectrogram (pitch angle averaged), a proton pitch angle spectrogram (energy averaged), an electron energy spectrogram (pitch angle averaged) and an electron pitch angle spectrogram (energy averaged) of 


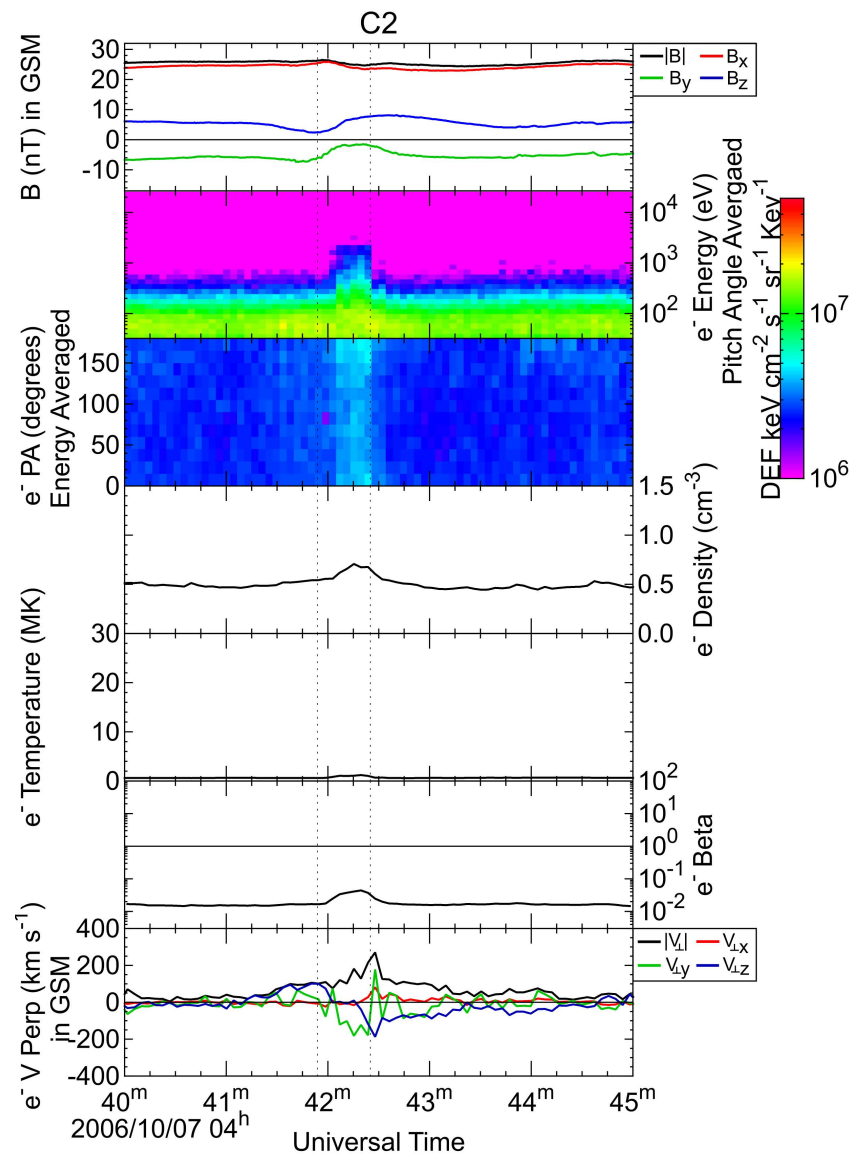

Fig. 7. $C 2$ observations. From top to bottom the panels shows the magnetic field strength ( $B_{X}$ is red, $B_{Y}$ is green, $B_{Z}$ is blue and $|B|$ is black), an electron energy spectrogram (pitch angle averaged), electron pitch angle spectrogram (energy averaged), electron density, electron temperature, electron $\beta$ and electron perpendicular velocity. Between 04:41-04:43 UT, the data shows little change in $B_{X}$ and $|B|$, a peak in $B_{Y}$ and a bipolar signature in $B_{Z}$. Simultaneously, an increase can be seen in the electron DEF, density and temperature. During the bipolar signature $\beta \sim 0.04$ indicating that the spacecraft is in the lobe at this time.

differential energy flux (in units of $\mathrm{keV} /\left(\mathrm{cm}^{2} \mathrm{~s} \mathrm{sr} \mathrm{keV}\right)$. Panels 6-9 show proton density, proton temperature, proton $\beta$ and proton perpendicular velocity. This spacecraft begins in the lobe (inferred from the proton $\beta$ ); at $\sim 04: 42$ UT it detects a negative-positive bipolar signature in $B_{Z}$. The peakto-peak amplitude is $\sim 18 \mathrm{nT}$. The first half of the bipolar signature shows more variability than the second. Coinciding with the bipolar signature, $B_{Y}$ peaks while $B_{X}$ and $|B|$ show significant dips of $\sim 9 \mathrm{nT}$ and $\sim 5 \mathrm{nT}$, respectively. The duration of the bipolar signature (again measured from peak to peak) is $\sim 29 \mathrm{~s}$, covering 145 magnetic field data points, 4 proton data points and 8 electron data points. During the bipolar signature, $\beta$ reaches 0.3 indicating that it is in the outer CPS/PSBL at this time. The proton energy and pitch angle spectrograms show two increases in DEF which coin-

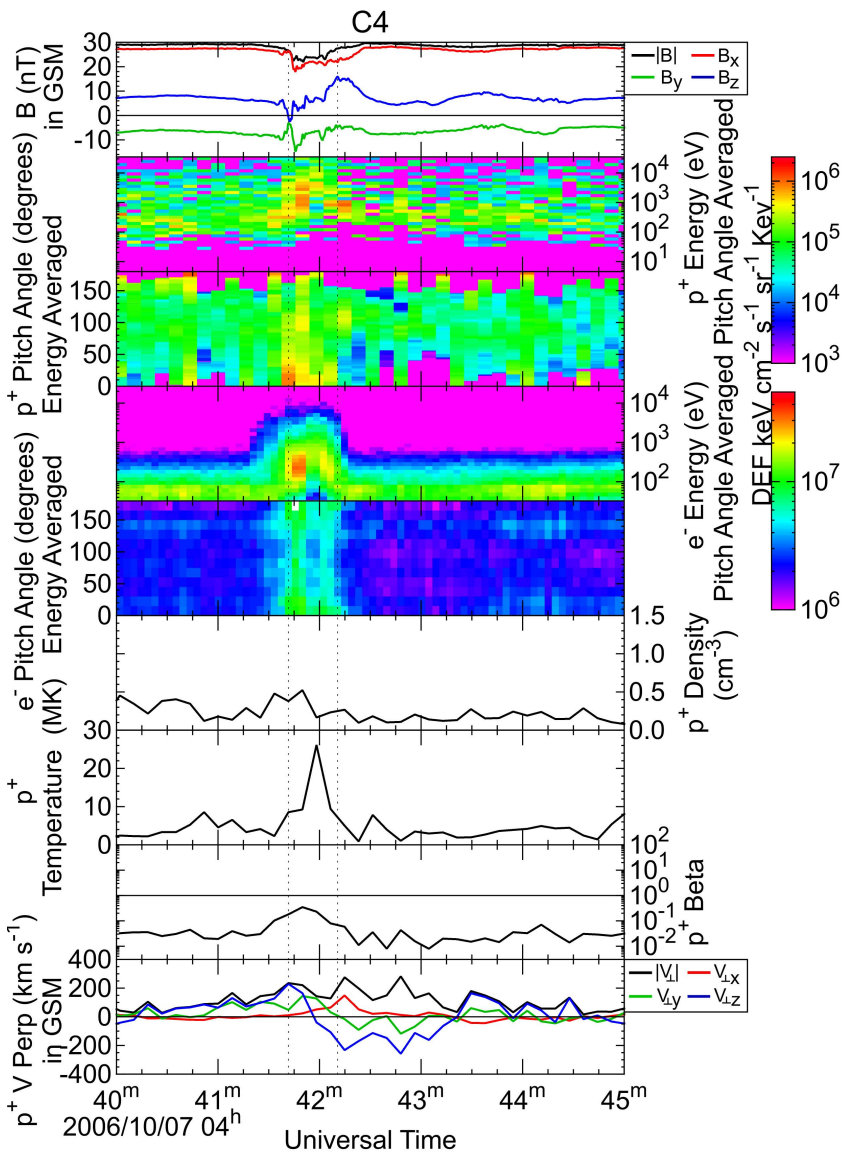

Fig. 8. $\mathrm{C} 4$ observations. From top to bottom the panels show magnetic field strength $\left(B_{X}\right.$ is red, $B_{Y}$ is green, $B_{Z}$ is blue and $|B|$ is black), proton energy spectrogram (pitch angle averaged), proton pitch angle spectrogram (energy averaged), electron energy spectrogram (pitch angle averaged), electron pitch angle spectrogram (energy averaged), proton density, proton temperature, proton $\beta$ and proton perpendicular velocity. Between 04:41-04:43 UT, C4 detected a dip in $B_{X}$ and $|B|$, a peak in $B_{Y}$ and a bipolar signature in $B_{Z}$. The spectrograms show two increases in DEF at the turning points of the bipolar signature; the increase in electron DEF was larger at the negative excursion than at the positive excursion. The increase in proton DEF was centred on a pitch angle of $0^{\circ}$. During the bipolar signature, $\beta \sim 0.3$ indicating that it was in the outer CPS/PSBL at this time.

cide with the turning points of the bipolar signature. They are centred on a pitch angle of $0^{\circ}$; the increase during the negative excursion has a greater pitch angle range and energy range compared to that observed during the positive excursion. The electron DEF also shows two increases at the turning points of the bipolar signature, the increase in DEF during the negative excursion is greater than that observed during the positive excursion. The pitch angle spectrogram shows the increase in electron DEF is greater for the $0^{\circ}$ and $180^{\circ}$ pitch angle electrons compared to the $90^{\circ}$ pitch angle electrons. The changes to the particle distributions also result 


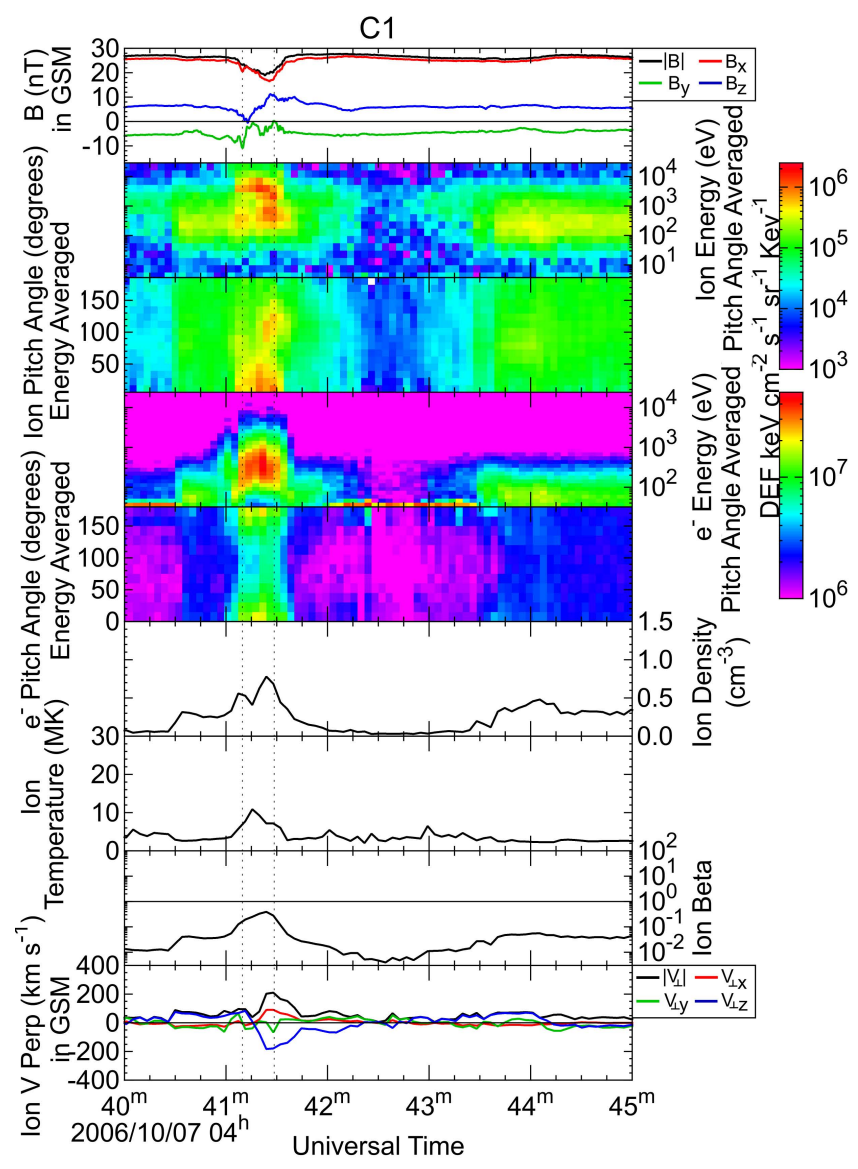

Fig. 9. $\mathrm{C} 1$ observations. From top to bottom the panels show magnetic field strength $\left(B_{X}\right.$ is red, $B_{Y}$ is green, $B_{Z}$ is blue and $|B|$ is black), ion energy spectrogram (pitch angle averaged), ion pitch angle spectrogram (energy averaged), electron energy spectrogram (pitch angle averaged), electron pitch angle spectrogram (energy averaged), ion density, ion temperature, ion $\beta$ and ion perpendicular velocity. Between 04:41-04:43 UT, C1 detects a dip in $B_{X}$, two dips in $B_{Y}$ and a bipolar signature in $B_{Z}$. The ion DEF increases at $0^{\circ}$ and between $100-1000 \mathrm{eV}$; simultaneously, two increases are seen between $1-10 \mathrm{keV}$ centred on $90^{\circ}$ and $110^{\circ}$. During the bipolar signature, $\beta \sim 0.4$ indicating that it was in the outer $\mathrm{CPS} / \mathrm{PSBL}$ at this time.

in accompanying increases in both the density and temperature; the density peaks during the negative excursion of the bipolar signature while the temperature peak coincides with the centre of the bipolar signature. The proton perpendicular velocity shows a north-south bipolar signature in $V_{\perp Z}$ and a peak in $V_{\perp X}$ of $\sim 150 \mathrm{~km} \mathrm{~s}^{-1}$.

Figure 9 displays the data from $\mathrm{C} 1$ in the same format as Fig. 8, except showing ion data in place of proton data. Initially, $\mathrm{C} 1$ is in the lobe (inferred from the proton $\beta$ ), at $\sim$ 04:40:30 UT it moves into a region of higher density lobe. At $\sim 04: 41: 20$ UT C1 detected a negative-positive bipolar signature in $B_{Z}$ with a peak-to-peak variation of $\sim 11 \mathrm{nT}$. The $B_{Y}$ component shows some variability and decreased from a background of $\sim-6 \mathrm{nT}$ to $\sim 0 \mathrm{nT}$ during two dips which closely coincide with the turning points of the bipolar signature. The $B_{X}$ component and $|B|$ show a reduction of $\sim 11 \mathrm{nT}$, with the minimum closely coinciding in time with the positive excursion of the bipolar $B_{Z}$ signature. The duration of the bipolar signature (again measured from peak to peak) is $\sim 19 \mathrm{~s}$, covering 93 magnetic field data points and 5 plasma data points. During the bipolar signature, $\beta$ reaches 0.4 indicating that it is in the outer CPS/PSBL at this time. Again, the magnetic variations are accompanied by changes in the particle distributions. At the turning points of the bipolar signature were two separate increases in ion DEF at energies between $200-1500 \mathrm{eV}$, which are centred on pitch angles of $\sim 90^{\circ}$ and $\sim 110^{\circ}$, respectively. The increase at the positive excursion of the bipolar signature covers a wider pitch angle range and has a larger ion DEF and duration compared to the increase at the negative excursion. Simultaneously, another increase in ion DEF, centred on $0^{\circ}$, can be seen at higher energies (between 1.5-32 keV); this increase is centred on the centre of the bipolar signature. During the bipolar signature, there was an increase in electron DEF at all pitch angles, which was greatest for the fieldaligned/anti-field aligned directions (100-1000 eV); the electron enhancements were also centred on the centre of the bipolar signature. Large electron DEFs were observed at low energies $(<50 \mathrm{eV})$ between 04:40:00-04:40:30 UT and 04:42:00-04:43:30 UT. However, these represent photoelectrons of spacecraft origin and are not an inherent feature of the magnetic structure. The ion density shows two separate peaks near the turning points of the bipolar signature. The second peak (which coincides with the positive excursion) was larger than the first, much like the increases in ion DEF. The ion temperature shows a peak caused by the higher energy ions seen in the spectrogram. The ion perpendicular velocity shows only moderate flows, with a north-south bipolar signature in $V_{\perp Z}$ and a peak in $V_{\perp X}$ at $\sim 100 \mathrm{~km} \mathrm{~s}^{-1}$.

Figure 10 contains the data from $\mathrm{C} 3$ in the same format as Fig. 8 except again with ion data instead of proton data. The C3 data shows a significantly different set of variations from those described above for the other 3 spacecraft. The spacecraft was in a region which is characterised by a relatively weak $(\sim 10 \mathrm{nT})$ and more variable magnetic field. Moreover, this spacecraft observes relatively high DEFs of ions (peaked near $1 \mathrm{keV}$ ) and electrons (peaked at a few hundred eV) and a high ion $\beta(>10)$ throughout the majority of this period, leading to generally higher densities and temperatures than those observed at the other spacecraft. These observations suggest that $\mathrm{C} 3$ was immersed in the CPS. Moreover at 04:42:30 UT, $B_{X}$ was close to zero, which suggests that the spacecraft was close to the neutral sheet at this time. Considering now the departures from this background, we note that there is no stand-out bipolar $B_{Z}$ signature in the $\mathrm{C} 3$ data during this period. Instead, a series of peaks at 04:41:45 UT, 04:43:15 UT and 04:44:00 UT, are observed in all 3 magnetic field components, which appear to vary closely in concert. 


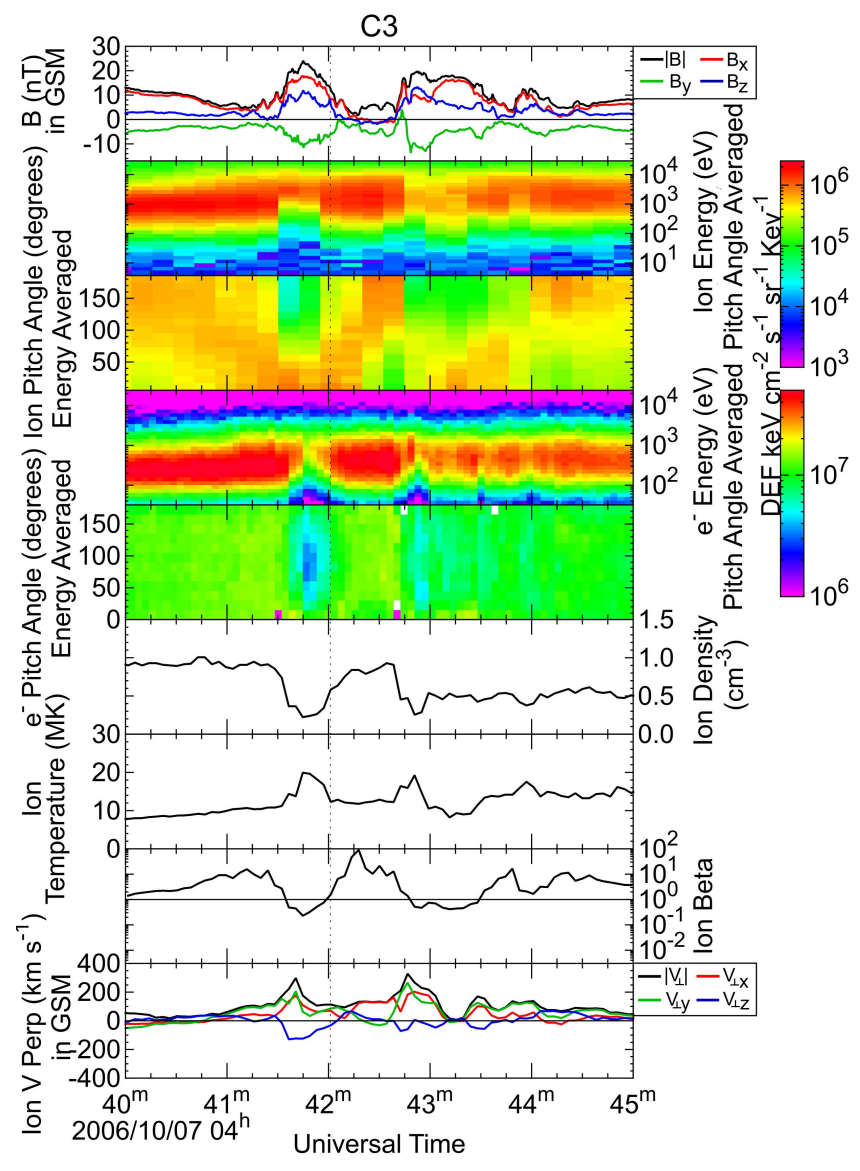

Fig. 10. $C 3$ observations. From top to bottom the panels show magnetic field strength ( $B_{X}$ is red, $B_{Y}$ is green, $B_{Z}$ is blue and $|B|$ is black), ion energy spectrogram (pitch angle averaged), ion pitch angle spectrogram (energy averaged), electron energy spectrogram (pitch angle averaged), electron pitch angle spectrogram (energy averaged), ion density, ion temperature, ion $\beta$ and ion perpendicular velocity. Across the time period, the magnetic field strength, temperature and perpendicular velocity exhibit a series of temporary increases, in conjunction with decreases in the particle DEF and density. The decreases in electron DEF is centred on $90^{\circ}$. Centred on 04:42:15 UT and 04:44:00 UT, the ion pitch angle spectrogram shows two rotations from $0^{\circ}$ to $180^{\circ}$. At 04:42:30 UT, $B_{X}$ was close to zero and $\beta>10$, which suggests that the spacecraft was deep in the central plasma sheet close to the neutral sheet at this time.

At the times of the magnetic maxima, there was a reduction of the ion and electron DEF, densities, $\beta$ (to $\sim 0.3$ which is outer CPS/PSBL) and an increase in ion temperature and ion perpendicular velocity. The reduction in electron DEF was predominantly in the direction perpendicular to the field. The ion pitch angle spectrogram (panel 3) shows two clear migrations between $0^{\circ}$ and $180^{\circ}$ centred on $04: 42: 15$ and 04:44:00 UT. For both rotations, while the pitch angle distribution is peaked at $0^{\circ}, B_{X}$ is the greatest component and while peaked at $180^{\circ}, B_{X}$ has reduced and $B_{Y}$ has increased. In both cases the variation in the directionality of the peak flux is not a full $180^{\circ}$ rotation in real space. After the magnetic variation ceased, the ion and electron DEFs and densities are reduced to below the pre-event levels.

\section{Analysis}

\subsection{Orientation of magnetic structure}

We now attempt to identify a co-ordinate system which may be aligned with any "quasi-invariant" axis of the structure responsible for the observations at each spacecraft which were described in the last section. We attempt to establish this coordinate system (which may also determine the orientation of the structure) by applying the minimum variance analysis (MVA) (Sonnerup and Cahill, 1967; Sonnerup and Scheible, 1998) on the magnetic field data from each spacecraft.

Minimum variance analysis performed on any vector dataset returns three eigenvectors (corresponding to the directions of the maximum, intermediate and minimum variance) and their associated eigenvalues (which are related to the size of the variation of the data components along the corresponding axis). The ratios of the intermediate/minimum and maximum/intermediate eigenvalues can be used as a measure of confidence in the determined axes (greater ratios indicate a greater confidence). A ratio of 10 is often used as the lowest acceptable accuracy (e.g. Paschmann and Daly, 1998). An eigenvalue ratio of 1 indicates that the associated eigenvectors are degenerate and that the uncertainty in their direction is thus $360^{\circ}$.

The results of MVA are somewhat subjective, and must be calculated and used with care. They are sensitive to the time interval across which the analysis is applied. For the event studied here, the greatest eigenvalue ratios tended to come from time limits imposed at the turning points of a bipolar signature as this is the region of greatest rate of change of magnetic field and direction. The turning points of the 3 bipolar signatures observed by $\mathrm{C} 1, \mathrm{C} 2$ and $\mathrm{C} 4$ also provide well-defined points seen in the all three magnetic signatures which facilitate comparisons between them.

As the C3 observations do not include an obvious bipolar signature and hence do not immediately match those of the other spacecraft, C3 data was not used in MVA calculations; the observations of $\mathrm{C} 2$ were also disregarded as this spacecraft passed outside of the structure. C1 and C4 both pass through the structure and hence the MVA calculations were based on the observations of these two spacecraft. The MVA axes were calculated using the time limits defined by the turning points of the bipolar signature, the average of the MVA $\mathrm{C} 1$ and $\mathrm{C} 4$ axes was then taken. The MVA axes of $\mathrm{C} 1$ were within $33^{\circ}$ of the equivalent $\mathrm{C} 4$ axes. The resulting MVA axes are as follows (in the format $\mathrm{X}$, $\mathrm{Y}, \mathrm{Z}$ in GSM): the vector of the minimum variance axis is $(0.886,-0.335,0.321)$, intermediate variance axis is $(0.379$, $0.897,-0.228)$, maximum variance axis is $(-0.205,0.288$, 
0.935). For comparison, the minimum, intermediate, maximum variance directions are loosely aligned $\left(<32^{\circ}\right)$ with $X$, Y, Z GSM, respectively.

\subsection{Velocity calculation}

Figure 1, 2, 3 and 4 show the bipolar signature is the largest magnetic variation (and hence is occurs in the maximum variance axis) and is perpendicular to the current sheet plane in both models. As mentioned previously, the flux rope and flux bulge both travel in the current sheet plane; hence the magnetic structure must be travelling in the plane perpendicular to the maximum variance axis (the minimumintermediate plane). If we assume that the magnetic structure was travelling at a constant speed and the distance between the spacecraft does not significantly change over the time periods of interest, we can calculate the velocity based on the encounter times and locations of the spacecraft. We expect the ratio of the time difference between the encounters of the magnetic structure with $\mathrm{C} 1$ and $\mathrm{C} 2$ and $\mathrm{C} 1$ and $\mathrm{C} 4$ to be the same as the ratio of the distances between the spacecraft in the minimum-intermediate plane. To calculate the direction of travel, the ratio of the distance between the spacecraft was calculated for all angles in the minimum-intermediate plane. The angle at which the distance ratio was closest to the time ratio was taken as the direction of travel. This was found to be $10^{\circ}$ anti-clockwise of the intermediate axis. The direction of travel agrees with the observed order in which the magnetic structure encounters the spacecraft $(\mathrm{C} 1, \mathrm{C} 4$, $\mathrm{C} 2$ ). Using the direction of travel, the locations and times, the magnetic structure was found to be moving at $(99,154$, -31) $|186| \mathrm{km} \mathrm{s}^{-1}$ GSM. Using this velocity, the encounter times and the locations of the spacecraft, the encounter time of the magnetic structure and $\mathrm{C} 3$ was estimated and found to be 04:42:01 UT. The intermediate variance direction was rotated by $10^{\circ}$ anti-clockwise about the maximum axis (to cause a rotation in the minimum-intermediate plane) to align the coordinate system with the direction of travel of the magnetic structure. The resulting MVA axes are as follows (in the format $\mathrm{X}, \mathrm{Y}, \mathrm{Z}$ in GSM): the vector of the minimum variance axis is $(0.801,-0.483,0.354)$, intermediate variance axis is $(0.531,0.830,-0.170)$, maximum variance axis is $(-0.205$, $0.288,0.935$ ). From here onwards, reference to the MVA axes refers to the rotated axes.

\subsection{Configuration in MVA derived coordinate system}

Figure 11 shows the spacecraft positions in the MVA coordinate system at 04:42:00 UT, a time close to that at which all three bipolar signatures were observed. The panels of the plot show the "minimum"-"maximum" plane in the top left, the "intermediate"-"maximum" plane in the top right and the "minimum"-"intermediate" plane in the bottom left. The spacecraft are coloured black, red, green and blue for $\mathrm{C} 1$, $\mathrm{C} 2, \mathrm{C} 3$ and $\mathrm{C} 4$, respectively.
As mentioned previously the maximum variance axis is perpendicular to the current sheet and hence the position of the spacecraft in this axis can hence be used as a proxy for the relative height of the spacecraft above the current sheet and thus the crossing height, or impact parameter, of the spacecraft through magnetic structure. The order in impact parameter deduced from the MVA coordinate system (from smallest to largest) is C3, C4, C1, C2. This agrees with the observations which show that C3 is in the CPS and that C2 observes a TCR indicating they have the lowest and highest impact parameter, respectively. In addition the observations show the enhancement in the electron DEF is greater at $\mathrm{C} 1$ than at $\mathrm{C} 4$, which is expected since $\mathrm{C} 1$ has a lower impact parameter than $\mathrm{C} 4$.

Conflicting with this, the observations also suggest $\mathrm{C} 1$ is at a higher impact parameter than $\mathrm{C} 4$. For example, initially $\mathrm{C} 1$ observes a lower density than $\mathrm{C} 4$; lower density regions are expected at the higher impact parameters. In addition, $\mathrm{C} 4$ has a larger (in peak-to-peak magnitude) bipolar signature compared to $\mathrm{C} 1$ which is expected at lower impact parameter crossings. The different plasma distributions may partly explain this disagreement; the positive excursion of the $\mathrm{C} 4$ bipolar signature has a lower diamagnetic reduction compared to that of $\mathrm{C} 1$, suggested by the lower electron DEF and hence density ( $\mathrm{C} 1$ 's ion spectrogram and moments cannot be directly compared with $\mathrm{C} 4$ 's proton spectrogram and moments). As the observations are somewhat inconclusive, we defer to the ordering calculated from the MVA coordinate system.

\subsection{Observations in MVA derived coordinate system}

Figure 12 shows data from all four spacecraft plotted in the MVA coordinate system. From top to bottom, the first two panels are of magnetic field strength and perpendicular particle velocity of $\mathrm{C} 2$, followed by the same for $\mathrm{C} 4, \mathrm{C} 1$ and $\mathrm{C} 3$, respectively. In each plot, the component in the minimum, intermediate and maximum variance direction is in red, green and blue respectively and the magnitude is in black. The perpendicular velocity of $\mathrm{C} 1$ and $\mathrm{C} 3$ is derived from the ions, $\mathrm{C} 4$ from the protons and $\mathrm{C} 2$ from the electrons. The plot includes dotted lines marking the turning points of the bipolar signature of $\mathrm{C} 1, \mathrm{C} 2$ and $\mathrm{C} 4$ and the estimate for the encounter time of the magnetic structure with $\mathrm{C} 3$.

Figure 12 also includes observations of the perpendicular velocity. In regions in which ideal MHD is a valid approximation, magnetic field lines are frozen into the plasma and hence they convect together. Particles may drift freely parallel to the field lines but not perpendicular and hence any perpendicular motion is indicative of the movement of the magnetic field lines and not just the particles; this can provide information on the convection of any underlying magnetic structure. Perpendicular particle velocity is defined as $V_{\perp}=\underline{b} \times \underline{V} \times \underline{b}$, where $\underline{b}$ is the unit magnetic field and $\underline{V}$ is 

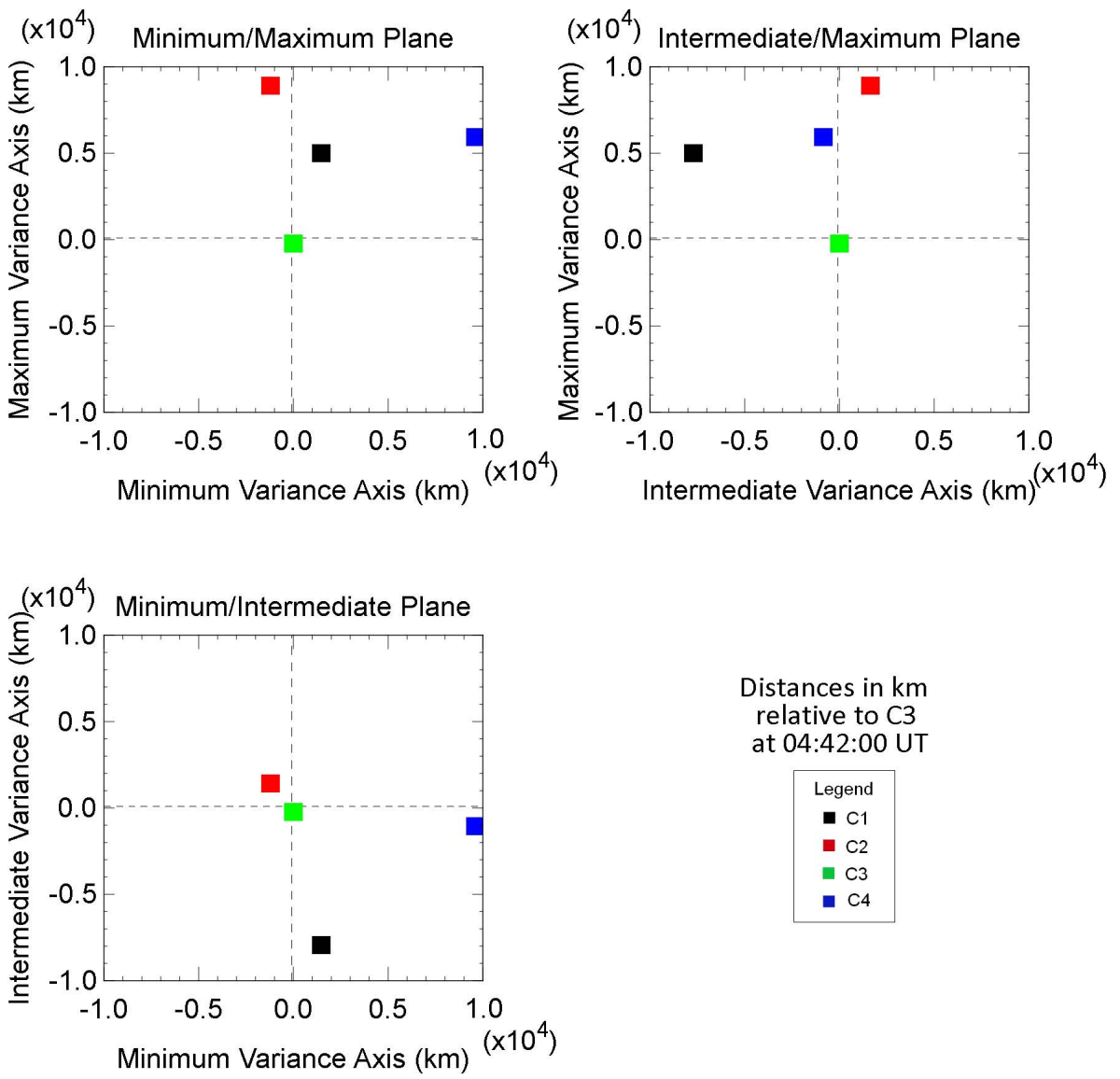

Fig. 11. Configuration of the spacecraft in the MVA co-ordinate system. C1, C2, C3 and C4 are in black, red, green and blue, respectively. The panels show the "minimum"-"maximum" plane in the top left, the "intermediate"-"maximum" plane in the top right and the "minimum""intermediate" plane in the bottom left. The direction of travel of the magnetic structure is along the intermediate variance direction; the plot shows the magnetic structure is expected to encounter the spacecraft in the order $\mathrm{C} 1, \mathrm{C} 4, \mathrm{C} 2$ which agrees with the observed times. The axis of impact parameter is parallel to the maximum axis. The order of the spacecraft in this axis is (from smallest to largest) $\mathrm{C} 3, \mathrm{C} 1, \mathrm{C} 4, \mathrm{C} 2$ which agrees with the observations.

the particle velocity. The data displayed in Fig. 12 are from the same data sources as Figs. 7-10.

Figure 12 shows the magnetic bipolar signature in this coordinate system is centred on zero indicating that the choice of maximum variance axis is accurate and perpendicular to the current sheet. The variations in $\mathrm{C} 3$ due to the reduction of the diamagnetic effect are mostly contained in $B_{\mathrm{MIN}}$ in this coordinate system. The estimated encounter time (04:42:01 UT) of the magnetic structure with C3 falls halfway between the first magnetic maximum (which coincides with PSBL-like plasma) and the magnetic minimum (04:41:30 UT, between the first two peaks, which coincides with CPS-like plasma). As both models predict a structure containing CPS-like plasma, we assume the CPS plasma observed at the $\mathrm{C} 3$ magnetic minimum is the plasma region associated with the magnetic structure. The plasma density at the C3 magnetic minimum was enhanced for $\sim 40$ s during the magnetic minimum, a period which, due to the resolu- tion of the instruments, covers 204 magnetic field data points and 10 plasma data points. Using this time and the velocity calculated previously, we estimate that the magnetic structure is $\sim 1.19 R_{\mathrm{E}}$ in size. As C3 is close to the neutral sheet whilst inside the magnetic structure, the minimum size of the magnetic structure (in the direction perpendicular to the current sheet) can be estimated by calculating the distance between the spacecraft close to its centre (C3) and the spacecraft with the highest impact parameter that crosses through the magnetic structure $(\mathrm{C} 4)$. The minimum size, from centre to edge, in the maximum variance direction is $\sim 0.97 R_{\mathrm{E}}$. As $\mathrm{C} 2$ is outside of the magnetic structure, the distance between it and $\mathrm{C} 3$ can be used as a maximum size, this is $\sim 1.43 R_{\mathrm{E}}$. Assuming that the structure is symmetrical north and south of the current sheet we obtain an estimate for the total size, from the outer edge on one side of the current sheet to the outer edge on the other side of the current sheet, this has a minimum of $\sim 1.94$ and a maximum of $\sim 2.86 R_{\mathrm{E}}$. 


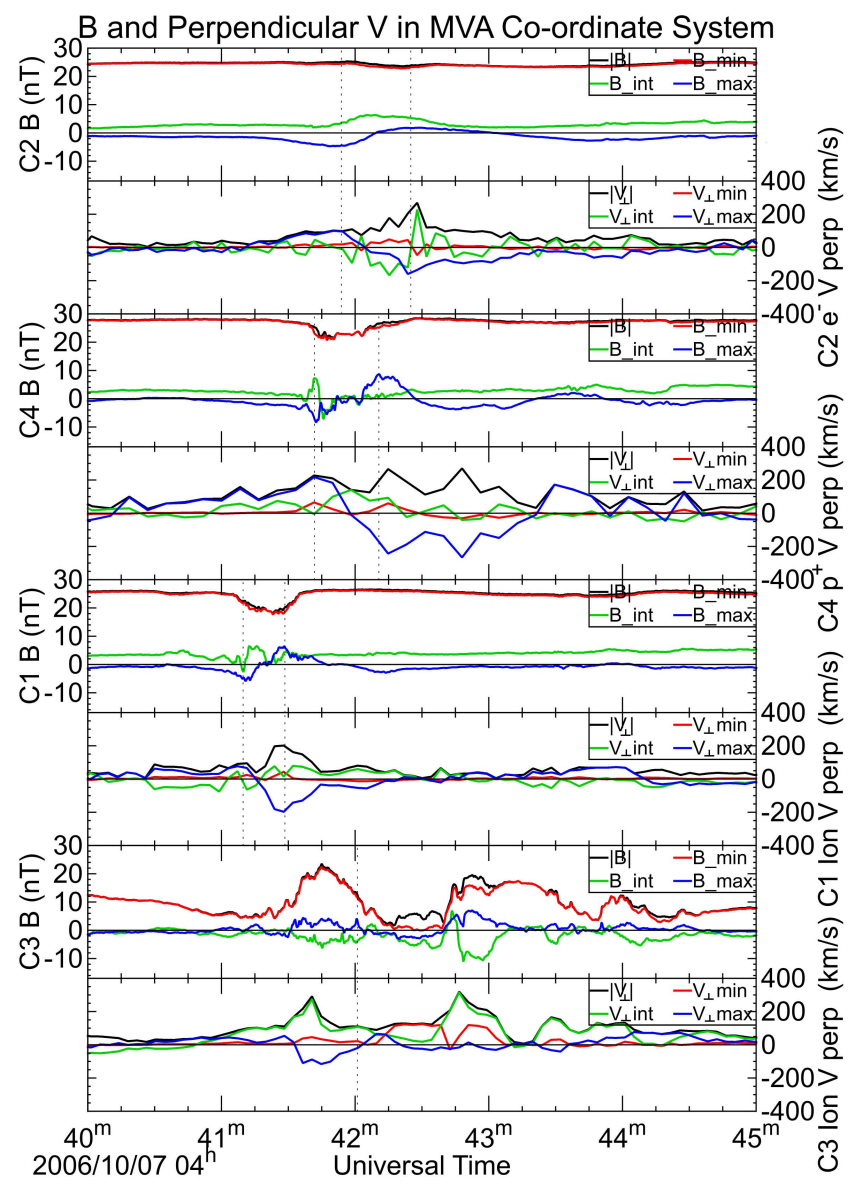

Fig. 12. Observations in the MVA co-ordinate system. The panels show from top to bottom the magnetic field strength and perpendicular velocity of $\mathrm{C} 2, \mathrm{C} 4, \mathrm{C} 1$ and $\mathrm{C} 3$. The magnitude is in black, the minimum, intermediate and maximum components are in red, green and blue, respectively. The dotted lines mark the turning points of the bipolar signature in all plots except $\mathrm{C} 3$ where it represents the estimated encounter time of the magnetic structure with $\mathrm{C} 3$. Between 04:41-04:43 UT, C1, C2 and C4 observe bipolar signatures in $B_{\mathrm{MAX}}$ which are centred on 0 . In $\mathrm{C} 3$ the variations occur mostly in $B_{\mathrm{MIN}}$.

The observed perpendicular velocity of the plasma at the centre of the magnetic structure (at the $\mathrm{C} 3$ magnetic minimum) is directed mostly in X (see Fig. 10). This differs from the calculated overall velocity which is travelling mostly in $\mathrm{Y}$. This can be explained by the direction of the field at the $\mathrm{C} 3$ magnetic minimum, which is mostly in $\mathrm{Y}$; movement in that direction is parallel to the field and hence does not contribute to the convective velocity of the structure.

As mentioned previously, two rotations can be seen in the pitch angle. During the first rotation, the magnitude of the magnetic field strength is low and hence small changes in the magnitude of the individual components leads to large changes in the magnetic field direction. Hence the rotation in pitch angle is more likely due to the changes in the magnetic field direction. In contrast the second rotation has a much greater magnetic field magnitude; in addition the magnetic field is directed mostly in the minimum variance direction yet there is little perpendicular velocity in this direction. Hence this is more likely a true reversal of the pitch angle of the particles which is an indication of the presence of an X-line.

One could argue that a bipolar signature can be seen in $\mathrm{C} 3$ centred on 04:42:40 UT, comprising of the magnetic minimum and the second magnetic maximum. A greater plasma DEF coincides with the negative excursion compared to the positive excursion, and this observation is also apparent at $\mathrm{C} 4$ indicating that the two signatures may align at this point. However, as the positive excursion of the possible bipolar signature of $\mathrm{C} 3$ coincides with an increase in magnetic field strength of all components, this is partly caused by a reduction in the diamagnetic effect caused by the concurrent reduction in plasma density. In addition the distribution of the plasma does not match that of $\mathrm{C} 1$, nor does it match the expected distribution of the plasma of the flux rope or flux bulge: in the case of the flux rope the distribution of the plasma is expected to be symmetric about the inflexion point of the bipolar signature; in the case of the flux bulge, a CPS-like density coincides with the positive excursion of the bipolar signature. Neither is seen at $\mathrm{C} 3$, which shows an asymmetric distribution of the plasma and a PSBL-like plasma at the positive excursion of the bipolar signature.

\section{Discussion}

\subsection{Interpretation of observations}

We have presented data recorded by the Cluster spacecraft on 7 October 2006 between 04:40:00 and 04:45:00 UT, when the 4 spacecraft were located in the magnetotail. Prior to the arrival of the magnetic structure, $\mathrm{C} 1, \mathrm{C} 2$ and $\mathrm{C} 4$ detect a plasma $\beta<0.3$; this indicates that the spacecraft are outside the CPS which is defined as plasma $\beta<0.3$ (Baumjohann et al., 1989). During the bipolar signature at $C 2$ the electron $\beta$ increases to $\sim 0.04$ which is outside of the CPS. During the bipolar signature the ion $\beta$ at $\mathrm{C} 1$ reaches $\sim 0.4$ and the proton $\beta$ at $\mathrm{C} 4$ reaches $\sim 0.3$; this indicates that both spacecraft moved into the outer CPS/PSBL. Conversely, C3 generally observes much larger values of $\beta$, often $\gg 0.3$, indicating that it remains mostly in the CPS for most of the period under consideration. For two brief periods either side of the magnetic minimum, plasma $\beta$ falls to $\sim 0.3$ and hence moves to the outer CPS/PSBL. During the magnetic minimum, C3 observed an ion $\beta \gg 10$, indicating that it was deep in the CPS.

As mentioned before, over a longer time period the plasma sheet is flapping; the possibility that this is the cause of the bipolar signature was ruled out earlier and the perpendicular velocity reinforces this. The drop-outs of plasma sheet plasma in the $\mathrm{C} 3$ observations could be due to an 
oscillation of the plasma sheet in the maximum variance direction; movement of the plasma sheet away from the spacecraft would cause it to exit the plasma sheet (the spacecraft would observe an increased $B$ due to the reduced diamagnetic effect). If this was the case, the change in plasma sheet direction of travel from south to north (reversal in $V_{\perp \mathrm{MAX}}$ ) would coincide with the peaks in $B$; but this is not seen in the observations.

Between 04:40 and 04:45 UT, C1, C2 and C4 each detected a bipolar signature in conjunction with an increase in plasma DEF, density, temperature and velocity, which are interpreted here as being due to the motion of a magnetic structure past the spacecraft and includes the displacement, over the spacecraft locations, of various plasma layers. The bipolar signature at $\mathrm{C} 2$ is smooth and relatively small in peak-topeak magnitude when compared to that observed at $\mathrm{C} 1$ and C4. The particle DEF, density, temperature and velocity increases seen during the bipolar signature are also smaller for $\mathrm{C} 2$ compared to $\mathrm{C} 1$ and $\mathrm{C} 4$. The $\mathrm{C} 2$ observation is consistent with a TCR signature (Slavin et al., 1992), formed by the draping and compression of the overlying magnetic field lines around the structure. $\mathrm{C} 2$ thus passed through the overlying disturbance while $\mathrm{C} 1$ and $\mathrm{C} 4$ appear to have passed through the structure itself.

The magnetic signature at $\mathrm{C} 2$ magnetic suggests that the draping signature is stronger in $B_{Y}$ than in $B_{Z}$, which may indicate that either the magnetic structure does not extend uniformly in both a duskward and dawnward direction relative to $\mathrm{C} 2$ and the spacecraft passes over the dusk "end" of the magnetic structure, or that the plasma sheet bulge was larger in diameter to the dawnward side of the spacecraft (e.g. Slavin et al., 1993, 1999).

Flux ropes and flux bulges are created both Earthwards and tailward of the (dominant) X-line and propagate away from the neutral line on each side. The direction of travel can be deduced from their observed velocities and by the nature of their bipolar signatures. The magnetic signatures reported here and observed by $\mathrm{C} 1, \mathrm{C} 2$ and $\mathrm{C} 4$ exhibited a negative $B_{Z}$ excursion followed by a positive $B_{Z}$ excursion, which is consistent with an Earthward travelling magnetic structure. In addition, the perpendicular velocities of $\mathrm{C} 1$ (ion), $\mathrm{C} 2$ (electron) and $\mathrm{C} 4$ (proton) show a $+V_{\perp X}$ GSM peak coinciding with the bipolar signature, which also indicates Earthward travel.

In common with the other spacecraft, C3 generally recorded a positive $B_{X}$ component of the magnetic field indicating that it mostly remained located north of the neutral sheet. We expect the lowest $B_{X}$ value in the magnetotail to be at the neutral sheet, which, assuming the magnetic structure is centred on the neutral sheet, corresponds to an impact parameter of zero. We note that $\mathrm{C} 3$ observed a $B_{X}$ value that is close to zero during the magnetic minimum which indicates that this spacecraft was located close to the neutral sheet at this time. Hence $\mathrm{C} 3$ underwent the centre-most crossing of the magnetic structure and would be expected to have ob- served the largest bipolar signature (a similar situation is reported in Walsh et al., 2007).

Instead of the expected bipolar signature, $\mathrm{C} 3$ observed a series of peaks in the magnetic field strength and temperature and a corresponding set of dips is seen in the density. This can be explained as a drop-out of the CPS plasma at the spacecraft location. Within these drop-outs, the observations are consistent with the PSBL, as indicated by a high magnetic field strength, low density and temperature and drop-outs of the perpendicular electron DEF. This situation is caused by $\mathrm{X}$-lines; once all of the CPS field lines are reconnected, the lower density PSBL field lines are reconnected next; a spacecraft would observe a CPS-like density followed by a PSBLlike density (this would be expected to occur in both modes of reconnection). The occurrence of reconnection is evident through the observation of parallel and anti-parallel streaming electrons seen at all spacecraft. The rotation of the ion pitch angles at the second drop-out may indicate that reconnection is occurring during the observation; since active Xlines expel plasma away from them on both sides, a spacecraft passing an active X-line would detect a reversal in the pitch angle of the particles. The first drop-out is also close to a rotation in pitch angle although this is thought to be due to the change in direction of the magnetic field; the drop-out is hence due to an $\mathrm{X}$-line that is no longer active (dormant $\mathrm{X}$-line).

Estimates of the expected encounter time of $\mathrm{C} 3$ return a result of 04:42:01 UT, which coincides with the boundary between the first drop-out in the CPS (dormant X-line) and the C3 magnetic minimum. Both models predict a structure with CPS-like plasma; therefore we assume CPS-like plasma of the $\mathrm{C} 3$ magnetic minimum is the plasma region of the magnetic structure.

We now compare the observations to the predictions of the two modes of reconnection.

\subsection{Multiple X-line reconnection interpretation}

A model of a flux rope which contains CPS-like plasma is able to readily explain the TCR signature seen at $\mathrm{C} 2$ and the bipolar signatures and plasma appearance at $\mathrm{C} 1$ and $\mathrm{C} 4$. However a flux rope, identifiable by a symmetrical bipolar signature with a symmetrical plasma distribution, is not seen in the $\mathrm{C} 3$ data. A possible explanation for this could be that the flux rope is early in it's development cycle, and does not yet have the structure that can be recognised as an observation of a quasi-force free flux rope. However, further consideration needs to be given to how such an 'early' flux rope would drive well developed signatures at the other spacecraft. Alternately, situations where X-lines are not parallel or are of greatly unequal lengths or do not have uniform reconnection rates along their length, may produce flux ropes with unusual edge effects, such that signatures matching the above description may not be observed at each of the 4 spacecraft in this case. 
In MXR, if two X-lines are present within the CPS and they begin reconnecting PSBL field lines, this leads to a situation of a CPS with two regions of PSBL-like plasma with a region of CPS-like plasma between them; this is consistent with the C3 observations. The TCR is caused by the increase in the pressure of the plasma between the X-lines due to the plasma outflow from the X-lines. The pressure of the tail perpendicular to the current sheet must be balanced; if the pressure of the plasma between the X-lines increases, it will expand to reduce its pressure and to re-establish pressure balance; hence this expansion causes the compression in the lobe and hence TCR observed at $\mathrm{C} 2$ and the observations at $\mathrm{C} 1$ and $\mathrm{C} 4$. In addition, MXR leads to one TCR from two $\mathrm{X}$-lines which is also consistent with the observations.

\subsection{Time dependant reconnection interpretation}

A model of a flux bulge which contains plasma-sheet-like plasma is able to readily explain the TCR signature seen at $\mathrm{C} 2$ and the bipolar signatures and plasma appearance at $\mathrm{C} 1$ and $\mathrm{C} 4$.

However the observations do not exhibit the predicted asymmetric bipolar signature with a CPS-like plasma density occurring concurrently with the positive excursion.

To create the two drop-outs in the plasma sheet downstream from a single $\mathrm{X}$-line, the reconnection rate would have to vary causing two bursts of reconnection. If the $\mathrm{X}$ line had reconnected all of the CPS field lines it would begin reconnecting PSBL field lines. If reconnection then slowed or ceased and then increased again at the same location, there would be no CPS field lines left and hence one would not expect to observe a CPS-like density between the two drop-outs seen in the observations.

\section{Conclusions}

We have presented multi-point observations of a TCR and the magnetic structure causing it, using data from the 4 Cluster spacecraft which each encounter the structure with different impact parameters. The estimated velocity of the structure, calculated from the timing of distinct signatures, is $(99,154$, $-31) \mathrm{km} \mathrm{s}^{-1}$ in GSM $\left(|\underline{V}|=186 \mathrm{~km} \mathrm{~s}^{-1}\right)$. The structure has an estimated size of $\sim 1.19 R_{\mathrm{E}}$ measured in the direction of travel and a size between of 1.94 and $2.86 R_{\mathrm{E}}$ measured in the direction perpendicular to the current sheet, assuming it is symmetrical about the current sheet. $\mathrm{C} 2$ passed outside of the structure and observed a TCR. C1 and C4 passed through the structure at high impact parameters and $\mathrm{C} 3$ passed through the centre. C3 observed a region of CPS-like plasma between two plasma sheet drop-outs. We believe the plasma sheet drop-outs are due to the passage of two X-lines past the spacecraft locations; concurrent with the second dropout, the spacecraft observes a rotation in the pitch angle of the ions from $0^{\circ}$ to $180^{\circ}$ which is consistent with the ex- pected signature of a reconnecting X-line. The occurrence of magnetic reconnection is also indicated by the observation of parallel/anti parallel electron fluxes. The TCR is caused by the increase in pressure and expansion of the plasma between the X-lines in the direction perpendicular to the current sheet. Although the observations do not fit in their entirety with the predictions of either the flux rope or the flux bulge models, the observation of two plasma sheet drop-outs (interpreted as $\mathrm{X}$-lines, one active, one dormant) with CPS-like plasma between them and only one TCR is evidence in favour of the multiple X-line reconnection model.

Acknowledgements. S. B. was supported by a UK STFC Ph.D. studentship. C. J. O., A. N. F., A. P. W. and C. F. O. were funded by the UK STFC under the UCL/MSSL Rolling Grant. S. K. acknowledges support by the Austrian "Fonds zur Förderung der wissenschaftlichen Forschung" under projects P20341-N16 and J3041N16. We acknowledge the work of the Cluster instrument teams and ESA Cluster Active Archive in preparing the data used in this study.

Topical Editor I. Daglis thanks two anonymous referees for their help in evaluating this paper.

\section{References}

Balogh, A., Carr, C. M., Acuña, M. H., Dunlop, M. W., Beek, T. J., Brown, P., Fornacon, K.-H., Georgescu, E., Glassmeier, K.H., Harris, J., Musmann, G., Oddy, T., and Schwingenschuh, K.: The Cluster Magnetic Field Investigation: overview of in-flight performance and initial results, Ann. Geophys., 19, 1207-1217, doi:10.5194/angeo-19-1207-2001, 2001.

Baumjohann, W., Paschmann, G., and Cattell, C. A.: Average Plasma Properties in the Central Plasma Sheet, J. Geophys. Res., 94, 6597-6606, doi:10.1029/JA094iA06p06597, 1989.

Biernat, H. K. and Heyn, M. F.: Unsteady Petschek reconnection, J. Geophys. Res., 92, 3392-3396, 1987.

Elphic, R. C., Cattell, C. A., Takahashi, K., Bame, S. J., and Russell, C. T.: ISEE-1 and 2 observations of magnetic flux ropes in the magnetotail: FTEs in the plasma sheet?, Geophys. Res. Lett., 13, 648-651, 1986.

Escoubet, C. P., Fehringer, M., and Goldstein, M.: Introduction The Cluster mission, Ann. Geophys., 19, 1197-1200, doi:10.5194/angeo-19-1197-2001, 2001.

Forsyth, C., Lester, M., Fear, R. C., Lucek, E., Dandouras, I., Fazakerley, A. N., Singer, H., and Yeoman, T. K.: Solar wind and substorm excitation of the wavy current sheet, Ann. Geophys., 27, 2457-2474, doi:10.5194/angeo-27-2457-2009, 2009.

Henderson, P. D., Owen, C. J., Alexeev, I. V., Slavin, J., Fazakerley, A. N., Lucek, E., and Rème, H.: Cluster observations of flux rope structures in the near-tail, Ann. Geophys., 24, 651-666, doi:10.5194/angeo-24-651-2006, 2006.

Hughes, W. J. and Sibeck, D. G.: On the 3-dimensional structure of plasmoids, Geophys. Res. Lett., 14, 636-639, 1987.

Imber, S. M., Slavin, J. A., Auster, H. U., and Angelopoulos, V.: A THEMIS survey of flux ropes and traveling compression regions: Location of the near-Earth reconnection site during solar minimum, J. Geophys. Res., 116, A02201, doi:10.1029/2010JA016026, 2011. 
Johnstone, A. D., Alsop, C., Burdge, S., Carter, P. J., Coates, A. J., Coker, A. J., Fazakerley, A. N., Grande, M., Gowen, R. A., Gurgiolo, C., Hancock, B. K., Narheim, B., Preece, A., heather, P. H., Winningham, J. D., and Woodcliffe, R. D.: PEACE, A Plasma Electron and Current Experiment, Space Sci. Rev., 79, 351-398, 1997.

Kiehas, S. A., Semenov, V. S., Kubyshkina, M. V., Angelopoulos, V., Nakamura, R., Keika, K., Ivanova, V. V., Biernat, H. K., Baumjohann, W., Mende, S., Magnes, W., Auster, U., Fornacon, K.-H., Larson, D., Carlson, C. W., Bonnell, J., and McFadden, J.: First Application of a Petschek-type Reconnection Model with Time-Varying Reconnection Rate to THEMIS Observations, J. Geophys. Res., 114, A00C20, doi:10.1029/2008JA013528, 2009.

Lee, L. C. and Fu, Z. F.: A theory of magnetic flux transfer at the Earth's magnetopause, Geophys. Res. Lett., 12, 105-108, doi:10.1029/GL012i002p00105, 1985.

Lepping, R. P., Jones, J. A., and Burlaga, L. F.: Magnetic Field Structure of Interplanetary Magnetic Clouds at 1 AU, J. Geophys. Res., 95, 11957-11965, 1990.

Moldwin, M. B. and Hughes, W. J.: Observations of Earthward and tailward propagating flux rope plasmoids: Expanding the plasmoid model of geomagnetic substorms, J. Geophys. Res., 99, 183-198, doi:10.1029/93JA02102, 1994.

Mulligan, T. and Russell, C. T.: Multispacecraft modeling of the flux rope structure of interplanetary coronal mass ejections: Cylindrically symmetric versus nonsymmetric topologies, J. Geophys. Res., 106, 10581-10596, doi:10.1029/2000JA900170, 2001.

Owen, C. J., Slavin, J. A., Fazakerley, A. N., Dunlop, M. W., and Balogh, A.: Cluster electron observations of the separatrix layer during traveling compression regions, Geophys. Res. Lett., 32, L03104, doi:10.1029/2004GL021767, 2005.

Paschmann, G. and Daly, P. (Eds.): Analysis Methods for MultiSpacecraft Data, ISSI Science Report, SR-001, 1998.

Petschek, H. E.: Magnetic field annihilation, AAS-NASA Symposium on the Physics of Solar Flares, NASA Spec. Publ. SP-50, 425-439, 1964.

Priest, E. R.: The equilibrium of magnetic flux ropes, Geophys. Monogr., 58, 1-22, 1990.

Rème, H., Aoustin, C., Bosqued, J. M., Dandouras, I., Lavraud, B., Sauvaud, J. A., Barthe, A., Bouyssou, J., Camus, Th., CoeurJoly, O., Cros, A., Cuvilo, J., Ducay, F., Garbarowitz, Y., Medale, J. L., Penou, E., Perrier, H., Romefort, D., Rouzaud, J., Vallat, C., Alcaydé, D., Jacquey, C., Mazelle, C., d’Uston, C., Möbius, E., Kistler, L. M., Crocker, K., Granoff, M., Mouikis, C., Popecki, M., Vosbury, M., Klecker, B., Hovestadt, D., Kucharek, H., Kuenneth, E., Paschmann, G., Scholer, M., Sckopke, N., Seidenschwang, E., Carlson, C. W., Curtis, D. W., Ingraham, C., Lin, R. P., McFadden, J. P., Parks, G. K., Phan, T., Formisano, V., Amata, E., Bavassano-Cattaneo, M. B., Baldetti, P., Bruno, R., Chionchio, G., Di Lellis, A., Marcucci, M. F., Pallocchia, G., Korth, A., Daly, P. W., Graeve, B., Rosenbauer, H., Vasyliunas, V., McCarthy, M., Wilber, M., Eliasson, L., Lundin, R., Olsen, S., Shelley, E. G., Fuselier, S., Ghielmetti, A. G., Lennartsson, W., Escoubet, C. P., Balsiger, H., Friedel, R., Cao, J.-B., Kovrazhkin, R. A., Papamastorakis, I., Pellat, R., Scudder, J., and Sonnerup, B.: First multispacecraft ion measurements in and near the Earth's magnetosphere with the identical Cluster ion spectrometry (CIS) experiment, Ann. Geophys., 19, 1303-1354, doi:10.5194/angeo19-1303-2001, 2001.

Runov, A., Sergeev, V. A., Baumjohann, W., Nakamura, R., Apatenkov, S., Asano, Y., Volwerk, M., Vörös, Z., Zhang, T. L., Petrukovich, A., Balogh, A., Sauvaud, J.-A., Klecker, B., and Rème, H.: Electric current and magnetic field geometry in flapping magnetotail current sheets, Ann. Geophys., 23, 1391-1403, doi:10.5194/angeo-23-1391-2005, 2005.

Schindler, K.: A Theory of the Substorm Mechanism, J. Geophys. Res., 79, 2803-2810, 1974.

Schödel, R., Baumjohann, W., Nakamura, R., Sergeev, V. A., and Mukai, T.: Rapid flux transport in the central plasma sheet, J. Geophys. Res., 106, 301-314, 2001.

Semenov, V. S., Heyn, M. F., and Kubyshkin, I. V., Reconnection of magnetic field lines in a nonstationary case, Sov. Astron., 27, 660-665, 1983.

Semenov, V. S., Vasilyev, E. P., and Purovkin, A. I., A scheme for the non-steady reconnection of magnetic lines of force, Geomagnet. Aeronomy (Engl. Transl.), 24, 370-373, 1984.

Sergeev, V. A., Tsyganenko, N. A., and Angelopoulos, V.: Dynamical response of the magnetotail to changes of the solar wind direction: an MHD modeling perspective, Ann. Geophys., 26, 2395-2402, doi:10.5194/angeo-26-2395-2008, 2008.

Slavin, J. A., Smith, M. F., Mazur, E. L., Baker, D. N., Iyemori, T., Singer, H. J., and Greenstadt, E. W.: ISEE 3 plasmoid and TCR observations during an extended interval of substorm activity, Geophys. Res. Lett., 19, 825-828, doi:10.1029/92GL00394, 1992.

Slavin, J. A., Smith, M. F., Mazur, E. L., Baker, D. N., Iyemori, T., and Greenstadt, E. W.: ISEE-3 Observations of Traveling Compression Regions in the Earth's Magnetotail, J. Geophys. Res., 98, 15425-15446, doi:10.1029/93JA01467, 1993.

Slavin, J. A., Owen, C. J., Kuznetsova, M. M., and Hesse, M.: ISEE 3 observations of plasmoids with flux rope magnectic topologies, Geophys. Res. Lett., 22, 2061-2064, doi:10.1029/95GL01977, 1995.

Slavin, J. A., Hesse, M., Owen, C. J., Taguchi, S., Fairfield, D. H., Lepping, R. P., Kokubun, S., Mukai, T., Lui, A. T. Y., Anderson, R. R., Matsumoto, H., and Sutcliffe, P. R.: Dual spacecraft observations of lobe magnetic field perturbations before, during and after plasmoid r elease, Geophys. Res. Lett., 26, 2897-2900, doi:10.1029/1999GL003606, 1999.

Slavin, J. A., Lepping, R. P., Gjerloev, J., Fairfield, D. H., Hesse, M., Owen, C. J., Moldwin, M. B., Nagai, T., Ieda, A., and Mukai, T.: Geotail observations of magnetic flux ropes in the plasma sheet, J. Geophys. Res., 108, 1015-1032, 2003a.

Slavin, J. A., Owen, C. J., Dunlop, M. W., Boralv, E., Moldwin, M. B., Sibeck, D. G., Transkanen, E., Goldstein, M. L., Fazakerley, A., Balogh, A., Lucek, E., Richter, I., Reme, H., and Bosqued, J. M.: Cluster four spacecraft measurements of small traveling compression regions in the near-tail, Geophys. Res. Lett., 30, 2208-2211, 2003b.

Slavin, J. A., Transkanen, E. I., Hesse, M., Owen, C. J., Dunlop, M. W., Imber, S., Lucek, E. A., Balogh, A., and Glassmier, K.-H.: Cluster observations of traveling compression regions in the near-tail, J. Geophys. Res., 110, A06207, doi:10.1029/2004JA010878, 2005.

Sonnerup, B. U. Ö. and Cahill, L. J.: Magnetopause structure and attitude from Explorer 12 observations, J. Geophys. Res., 72, 
171-183, 1967.

Sonnerup, B. U. Ö. and Scheible, M.: Minimum and maximum variance analysis, in: Analysis Methods for Multi-Spacecraft Data, edited by: Paschmann, G. and Daly, P., ESA, Noordwijk, 185-220, 1998.

Walsh, A. P., Fazakerley, A. N., Wilson, R. J., Alexeev, I. V., Henderson, P. D., Owen, C. J., Lucek, E., Carr, C., and Dandouras, I.: Near-simultaneous magnetotail flux rope observations with Cluster and Double Star, Ann. Geophys., 25, 1887-1897, doi:10.5194/angeo-25-1887-2007, 2007.
Zhang, T. L., Baumjohann, W., Nakamura, R., Balogh, A., and Glassmeier, K.-H.: A wavy twisted neutral sheet observed by CLUSTER, Geophys. Res. Lett., 29, 5-1, 2002.

Zong, Q.-G., Fritz, T. A., Pu, Z. Y., Fu, S. Y., Baker, D. N., Zhang, H., Lui, A. T., Vogiatzis, I., Glassmeier, K.-H., Korth, A., Daly, P. W., Balogh, A., and Reme, H.: Cluster observations of Earthward flowing plasmoid in the tail, Geophys. Res. Lett., 31, L18803, doi:10.1029/2004GL020692, 2004. 